\title{
Maximizing the Geometric Mean of User-Rates to Improve Rate-Fairness: Proper vs. Improper Gaussian Signaling
}

\author{
H. Yu ${ }^{1}$, H. D. Tuan ${ }^{1}$, E. Dutkiewicz ${ }^{1}$, H. V. Poor ${ }^{2}$, and L. Hanzo ${ }^{3}$
}

\begin{abstract}
This papers considers a reconfigurable intelligent surface (RIS)-aided network, which relies on a multiple antenna array aided base station (BS) and a RIS for serving multiple single antenna downlink users. To provide reliable links to all users over the same bandwidth and same time-slot, the paper proposes the joint design of linear transmit beamformers and the programmable reflecting coefficients of an IRS to maximize the geometric mean (GM) of the users' rates. A new computationally efficient alternating descent algorithm is developed, which is based on closed-forms only for generating improved feasible points of this nonconvex problem. We also consider the joint design of widely linear transmit beamformers and the programmable reflecting coefficients to further improve the GM of the users' rates. Hence another alternating descent algorithm is developed for its solution, which is also based on closed forms only for generating improved feasible points. Numerical examples are provided to demonstrate the efficiency of the proposed approach.
\end{abstract}

Index Terms-Reconfigurable intelligent surface, proper and improper Gaussian signaling, transmit beamforming, trigonometric function optimization, geometric mean maximization, nonconvex optimization algorithms

\section{INTRODUCTION}

The spectral efficiency optimization of wireless networks is often carried out by sum rate (SR) maximization, thanks to the computational tractability of the latter when relying on beamforming [1], [2]. However, by its nature, SR maximization has the deficiency of allocating a large fraction of the sum-rate to a few users having good channel conditions, while leaving the rest of the users with almost zero rates. Furthermore, the SR performance is typically improved with more users involved because there are more flexible choices for the users' channels [3]. The spectral efficiency is thus addressed more appropriately via either SR maximization under specific quality-of-service (QoS) constraints in terms of the users' minimum rate, or by max-min user-rate optimization, but their computation is quite demanding [1], [2], [4]-[6].

Reconfigurable intelligent surfaces (RISs) [7] are constructed by a planar array of programmable reflecting elements

This work was supported in part by the Australian Research Councils Discovery Projects under Grant DP190102501, and in part by the Engineering and Physical Sciences Research Council projects EP/P034284/1 and EP/P003990/1 (COALESCE), and European Research Council's Advanced Fellow Grant QuantCom (Grant No. 789028)

${ }^{1}$ School of Electrical and Data Engineering, University of Technology Sydney, Broadway, NSW 2007, Australia (email:Hongwen.Yu@student.uts.edu.au, Tuan.Hoang@uts.edu.au, Eryk.Dutkiewicz@uts.edu.au); ${ }^{2}$ Department of Electrical and Computer Engineering, Princeton University, Princeton, NJ 08544, USA (email: poor@princeton.edu); ${ }^{3}$ School of Electronics and Computer Science, University of Southampton, Southampton, SO17 1BJ, UK (email: lh@ecs.soton.ac.uk)
(PREs), which have recently been introduced for improving the energy and spectral efficiencies of future wireless networks (6G) [8]-[11], the coverage, reliability and the average achievable rate of UAV communication systems [12]-[14] and the outage probability and bit error rate (BER) of indoor mixed dual-hop VLC/RF systems [15]. Moreover, channel estimation and physical layer security for RIS-aided networks have been studied recently [16]-[19]. A typical RIS-aided system consists of a base station (BS) and a RIS for beneficially reflecting the incident electromagnetic waves from the $\mathrm{BS}$ to multi-target directions, where the spectral efficiency may be improved by the joint design of the transmit beamformer at the BS and RIS PREs [20]. The joint design is often based on alternating optimization between the beamformer and PREs. Thus, compared to the design of stand-alone transmit beamformers, the new challenge is the optimization of the PREs with the beamformer weights fixed, which is computationally challenging due to the nonconvex unit-modulus constraint (UMC) imposed on the PREs. In [8] and [21], general-purpose gradient/projected gradient algorithms were used, which do not necessarily converge. By contrast to either convex relaxation relying on dropping the matrix-rank of one constraint or on relaxing the UMC to the convex bounded-by-unit-modulus constraint were used in [22]-[26] for mitigating the computational challenge. Except for the works [22] and [26], which particularly considered the problem of transmit power minimization subject to signal-to-interference-plus-noise ratio (SINR) constraints, all the following treatises [8], [21], [23][25] considered the problem of SR maximization. The authors of [23]-[25] applied convex relaxation not only to the UMC but also to the SR objective function. It should be noted that alternating optimization between two sets of decision variables is only efficient, when the optimization within each set with the other set held fixed is computationally tractable. However that is not the case for the problems considered in all these papers because both the optimization of the beamformers with the PREs held fixed and that of the PREs with the beamformer weights held fixed present difficult nonconvex problems. In the end, the convergence of alternating optimization-based algorithms to a locally optimal solution is not guaranteed. Our recent work [27] has been the first one that addressed the spectral efficiency of RIS-aided communication via maxmin user-rate optimization. Instead of alternating optimization, we proposed an alternating descent at the first stage and then a joint descent at the second stage to confirm the optimality of the solutions computed. While the descent iterations in the 
beamformers generate a sequence of better feasible points, the descent iterations in the PREs generate a sequence of better infeasible points, which converges to a feasible point. Moreover, it has been also shown in [27] that using widely linear beamformers for facilitating improper Gaussian signaling (IGS) improves the users' max-min rate. To sum up, we provide a brief comparison of the related literature in Table I.

Against the above background, this paper offers the following contributions:

- We consider the problem of maximizing the geometric mean (GM) of users' rates for allocating the rates to all users in an equitable manner. We use the users' rate deviation (RD) from their mean and the ratio of the users' maximal and minimal rates (RR) as the main criterion to judge the users' rate balance, which are 0 and 1 , respectively, when all users are granted the same rate. The smaller these values are, the fairer the users' rate allocation becomes (more balanced).

- As this problem of GM maximization is computationally intractable, we address it via the min-max joint design of beamforming weights and RIS PREs. To eliminate the UMC of the RIS PREs, we use the polar form of unit-modulus complex numbers that allows each descent iteration of the RIS coefficient calculation to be based on the closed-form solution of an unconstrained nonconvex problem in the PREs' arguments. Each descent iteration of the beamformer weights and the PREs' arguments are also based on the closed-form solutions of convex problems. Thus, the proposed alternating descent method is purely based on closed forms and hence it is computationally efficient.

- Like in [27], here we also use improper Gaussian signaling (IGS) in the BS signal transmission, which has been shown to substantially improve the users' max-min rates (see e.g. [28]-[32]) thanks to its ability to mitigate the severe interferences in interference-limited systems. The performance gap between IGS and conventional proper Gaussian signaling (PGS) becomes substantially wider under more severe interference regimes. To elaborate a little further, IGS is not useful in interference-free regimes such as that of zero-forcing beamforming, which forces all interferences to zero. The interference scenario of SR maximization under PGS is unique in the sense that those users who were allocated zero-rate impose no interference on the other users. As a result, SR maximization under PGS exhibit a high RD and nearinfinite RR. Our finding is that compared to PGS, IGS does not improve the system's SR but it results in much lower RD and RR as a benefit of having no users with zero rate. Hence SR maximization becomes a practically feasible option while providing the users with beneficial rate-fairness.

The paper is organized as follows. The joint design of beamformer weights and PREs to maximize the GM of users' rates by tractable computation both under PGS and IGS is addressed in Section II and III, respectively. Their performances are evaluated by the simulations in Section IV, while Section V concludes the paper.

Notation. Only the vector/matrix variables are printed in boldface; $I_{N}$ is the identity matrix of size $N \times N$, while $O_{M \times N}$ is a zero matrix of size $M \times N$. For $x=$ $\left(x_{1}, \ldots, x_{n}\right)^{T}, \operatorname{diag}(x)$ is a diagonal matrix of the size $n \times n$ with $x_{1}, x_{2}, \ldots, x_{n}$ on its diagonal; $[X]^{2}$ is $X X^{H}$, and $\langle X, Y\rangle=\operatorname{trace}\left(X^{H} Y\right)$ for the matrices $X$ and $Y$. Accordingly, the Frobenius norm of $X$ is defined by $\|X\|=$ $\sqrt{\operatorname{trace}\left(X^{H} X\right)}$. We also write $\langle X\rangle=\operatorname{trace}(X)$ for notational simplicity. The notation $X \succeq 0$ ( $X \succ 0$, resp.) used for the Hermitian symmetric matrix $X$ indicates that it is positive definite (positive semi-definite, resp.). Let us denote the maximal eigenvalue of the Hermitian symmetric matrix $X$ by $\lambda_{\max }(X) ; \operatorname{vec}(X)$ stacks the columns of the matrix $X$ into a single column (vector) and as such we have $\operatorname{vec}(A X B)=\left(B^{T} \otimes A\right) \operatorname{vec}(X)$ for the matrices $A$, $X$, and $B$ of appropriate sizes, where $\otimes$ is the Kronecker product. For a real valued vector $x=\left(x_{1}, \ldots, x_{n}\right)^{T} \in \mathbb{R}^{n}$, $e^{\jmath x}, \cos x$, and $\sin x$ are entry-wise understood, i.e. $e^{\jmath x}=$ $\left(e^{\jmath x_{1}}, \ldots, e^{\jmath x_{n}}\right)^{T} \in \mathbb{C}^{n}, \cos x=\left(\cos x_{1}, \ldots, \cos _{n}\right)^{T} \in \mathbb{R}^{n}$, and $\sin x=\left(\sin x_{1}, \ldots, \sin x_{n}\right)^{T} \in \mathbb{R}^{n}$. As such $e^{\jmath x}=$ $\cos x+\jmath \sin x$. For a complex number $x, \angle x$ denotes its argument, i.e. $x=e^{\jmath \angle x}$ for $|x|=1$ and it is fully characterized by $\angle x \in[0,2 \pi]$. Lastly, let us denote the set of circular Gaussian random variables with the zero means and variance $a$ by $\mathcal{C}(0, a)$. Each $s \in \mathcal{C}(0, a)$ is termed as being proper because $\mathbb{E}\left(s^{2}\right)=\mathbb{E}\left(\Re^{2}\{s\}\right)-\mathbb{E}\left(\Im^{2}\{s\}\right)=0$ as $\mathbb{E}\left(\Re^{2}\{s\}\right)=$ $\mathbb{E}\left(\Im^{2}\{s\}\right)=a / 2$. By contrast, a Gaussian random vector variable $x$ is referred to as improper if $\mathbb{E}\left(x x^{T}\right) \neq 0$, which particularly implies that $\mathbb{E}\left(\Re\{x\} \Re^{T}\{x\}\right) \neq \mathbb{E}\left(\Im\{x\} \Im^{T}\{x\}\right)$.

\section{PROPER GAUSSIAN SIGNALING}

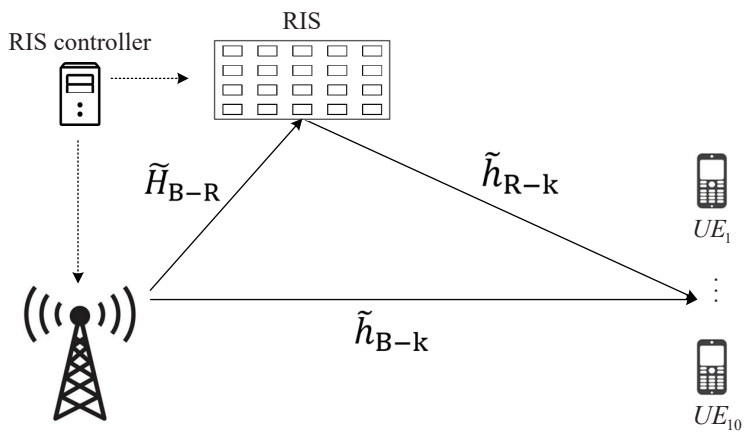

Fig. 1: System model

We consider the RIS-aided communication system illustrated by Fig. 1, where a RIS of $N$ reflecting units supports the downlink spanning from an $M$-antenna array BS to $K$ single-antenna users (UEs) $k \in \mathcal{K} \triangleq\{1, \ldots, K\}$. Since the RIS is typically deployed on the facade of high-rise buildings and the AP is also usually at a certain elevated height [10], it is justified to assume a LoS link between the AP and RIS, LoS communication between the RIS and UEs, and NLoS propagation between the AP and UEs. Accordingly, the channels spanning from the BS and the RIS to UE $k$ and from the BS to the RIS are modelled by $\tilde{h}_{\mathrm{B}-\mathrm{k}}=\sqrt{\beta_{\mathrm{B}-\mathrm{k}}} h_{\mathrm{B}-\mathrm{k}} \in \mathbb{C}^{1 \times M}$, 
TABLE I: A brief comparison of the related literature.

\begin{tabular}{|l|c|c|c|c|c|c|c|c|c|}
\hline Literature & This work & {$[27]$} & {$[8]$} & {$[21]$} & {$[22]$} & {$[23]$} & [24] & [25] & [26] \\
\hline SR maximization & $\sqrt{ }$ & & $\sqrt{ }$ & $\sqrt{ }$ & & $\sqrt{ }$ & $\sqrt{ }$ & $\sqrt{ }$ & \\
\hline max-min rate optimization & & $\sqrt{ }$ & & & & & & & \\
\hline GM maximization & $\sqrt{ }$ & & & & & & & & \\
\hline power minimization & & & & & $\sqrt{ }$ & & & & $\sqrt{ }$ \\
\hline PGS (linear beamforming) & $\sqrt{ }$ & $\sqrt{ }$ & $\sqrt{ }$ & $\sqrt{ }$ & $\sqrt{ }$ & $\sqrt{ }$ & $\sqrt{ }$ & $\sqrt{ }$ & $\sqrt{ }$ \\
\hline IGS (widely linear beamforming) & $\sqrt{ }$ & $\sqrt{ }$ & & & & & & & \\
\hline trigonometric function optimization & $\sqrt{ }$ & & & & & & & & \\
\hline computational tractability in PREs & $\sqrt{ }$ & $\sqrt{ }$ & & & & & & & \\
\hline
\end{tabular}

$\tilde{h}_{\mathrm{R}-\mathrm{k}}=\sqrt{\beta_{\mathrm{R}-\mathrm{k}}} h_{\mathrm{R}-\mathrm{k}} \in \mathbb{C}^{1 \times N}$, and $\tilde{H}_{\mathrm{B}-\mathrm{R}}=\sqrt{\beta_{\mathrm{B}-\mathrm{R}}} H_{\mathrm{B}-\mathrm{R}} \in$ $\mathbb{C}^{N \times M}$, where $\sqrt{\beta_{\mathrm{B}-\mathrm{k}}}, \sqrt{\beta_{\mathrm{R}-\mathrm{k}}}$, and $\sqrt{\beta_{\mathrm{B}-\mathrm{R}}}$ model the path-loss and large-scale fading of the BS-to-UE $k$ link, the RIS-to-UE $k$ link, and the BS-to-RIS link, respectively [21], [33], while $h_{\mathrm{R}-\mathrm{k}}$ and $H_{\mathrm{B}-\mathrm{R}}$ are modelled by Rician fading for modeling the line-of-sight (LoS) channels between the RIS and the UEs as well as between the BS and the RIS [34]. Furthermore, $h_{\mathrm{B}-\mathrm{k}}$ is modelled by Rayleigh fading in the face of non-LoS (NLoS) channels between the BS and the UEs. Like many other papers on RIS-aided communication networks, we assume having perfect channel state information, which can be obtained from channel estimation [8], [16], [22].

Let $s_{k} \in \mathcal{C}(0,1)$ be the information symbol intended for UE $k$, which is beamformed by $\mathbf{w}_{k} \in \mathbb{C}^{M}$. The signal $x$ transmited from the BS is

$$
x=\sum_{k \in \mathcal{K}} \mathbf{w}_{k} s_{k} .
$$

The signal received at UE $k$ can be expressed as

$$
\begin{aligned}
y_{k} & =\left(\tilde{h}_{\mathrm{R}-\mathrm{k}} \mathbf{R}_{\mathrm{R}-\mathrm{k}}^{1 / 2} \operatorname{diag}\left(e^{\jmath \boldsymbol{\theta}}\right) \tilde{H}_{\mathrm{B}-\mathrm{R}}+\tilde{h}_{\mathrm{B}-\mathrm{k}}\right) x+n_{k} \\
& =\mathcal{H}_{k}(\boldsymbol{\theta}) \sum_{k \in \mathcal{K}} \mathbf{w}_{k} s_{k}+n_{k},
\end{aligned}
$$

for

$$
\mathcal{H}_{k}(\boldsymbol{\theta}) \triangleq \tilde{h}_{\mathrm{BR}-\mathrm{k}} \operatorname{diag}\left(e^{\jmath \boldsymbol{\theta}}\right) H_{\mathrm{B}-\mathrm{R}}+\tilde{h}_{\mathrm{B}-\mathrm{k}} \in \mathbb{C}^{1 \times M},
$$

with

$$
\tilde{h}_{\mathrm{BR}-\mathrm{k}} \triangleq \sqrt{\beta_{\mathrm{B}-\mathrm{R}}} \sqrt{\beta_{\mathrm{R}-\mathrm{k}}} h_{\mathrm{R}-\mathrm{k}} \mathbf{R}_{\mathrm{R}-\mathrm{k}}^{1 / 2} \in \mathbb{C}^{1 \times N},
$$

where $\mathbf{R}_{\mathrm{R}-\mathrm{k}} \in \mathbb{C}^{N \times N}$ represents the spatial correlation matrix of the RIS elements with respect to user $k$ [21], [35], $n_{k} \in$ $\mathcal{C}(0, \sigma)$ is the background noise at UE $k$, and $\operatorname{diag}\left(e^{\boldsymbol{\theta} \boldsymbol{\theta}}\right)$ in (2) for $\boldsymbol{\theta}=\left(\boldsymbol{\theta}_{1}, \ldots, \boldsymbol{\theta}_{N}\right)^{T} \in[0,2 \pi]^{N}$ represents the matrix of PREs.

Let $\mathbf{w} \triangleq\left\{\mathbf{w}_{k}, k \in \mathcal{K}\right\}$. The rate in nats/sec at $\mathrm{UE} k$ is

$$
r_{k}(\mathbf{w}, \boldsymbol{\theta})=\ln \left(1+\frac{\left|\mathcal{H}_{k}(\boldsymbol{\theta}) \mathbf{w}_{k}\right|^{2}}{\sum_{j \in \mathcal{K} \backslash\{k\}}\left|\mathcal{H}_{k}(\boldsymbol{\theta}) \mathbf{w}_{j}\right|^{2}+\sigma}\right) .
$$

We consider the following problem of jointly designing the beamformers' weight set $\mathbf{w}$ and the PREs $\boldsymbol{\theta}$ to maximize the GM of users' rates:

$$
\begin{array}{r}
\max _{\mathbf{w}, \boldsymbol{\theta}}\left(\prod_{k=1}^{K} r_{k}(\mathbf{w}, \boldsymbol{\theta})\right)^{1 / K} \text { s.t. } \\
\sum_{k=1}^{K}\left\|\mathbf{w}_{k}\right\|^{2} \leq P
\end{array}
$$

where (7b) sets the transmit power constraint within a given power budget $P$. It is plausible that this problem is equivalent to the following one:

$$
\begin{array}{r}
\min _{\mathbf{w}, \boldsymbol{\theta}} f\left(r_{1}(\mathbf{w}, \boldsymbol{\theta}), \ldots, r_{K}(\mathbf{w}, \boldsymbol{\theta})\right) \triangleq \frac{1}{\left(\prod_{k=1}^{K} r_{k}(\mathbf{w}, \boldsymbol{\theta})\right)^{1 / K}} \\
\text { s.t. } \quad(7 b) .
\end{array}
$$

The function $f\left(r_{1}(\mathbf{w}, \boldsymbol{\theta}), \ldots, r_{K}(\mathbf{w}, \boldsymbol{\theta})\right)$ is the composition of the convex function $f\left(r_{1}, \ldots, r_{K}\right)=1 /\left(\prod_{k=1}^{K} r_{k}\right)^{1 / K}$ and the non-convex functions $r_{k}(\mathbf{w}, \boldsymbol{\theta}), k=1, \ldots, K$.

Let $\left(w^{(\kappa)}, \theta^{(\kappa)}\right)$ be a feasible point for (8) that is found from the $(\kappa-1)$-st round. We note that the linearized function of $f\left(r_{1}(\mathbf{w}, \boldsymbol{\theta}), \ldots, r_{K}(\mathbf{w}, \boldsymbol{\theta})\right)$ at $\left(r_{1}\left(w^{(\kappa)}, \theta^{(\kappa)}\right), \ldots, r_{K}\left(w^{(\kappa)}, \theta^{(\kappa)}\right)\right)$ is

$$
\begin{array}{r}
2 f\left(r_{1}\left(w^{(\kappa)}, \theta^{(\kappa)}\right), \ldots, r_{K}\left(w^{(\kappa)}, \theta^{(\kappa)}\right)\right)-f\left(r_{1}\left(w^{(\kappa)}, \theta^{(\kappa)}\right)\right. \\
\left.\ldots, r_{K}\left(w^{(\kappa)}, \theta^{(\kappa)}\right)\right) \frac{1}{K} \sum_{k=1}^{K} \frac{r_{k}(\mathbf{w}, \boldsymbol{\theta})}{r_{k}\left(w^{(\kappa)}, \theta^{(\kappa)}\right)} .
\end{array}
$$

Since we have $f\left(r_{1}\left(w^{(\kappa)}, \theta^{(\kappa)}\right), \ldots, r_{K}\left(w^{(\kappa)}, \theta^{(\kappa)}\right)\right)>0$, we can use steepest descent optimization for the convex function $f\left(r_{1}, \ldots, r_{K}\right)$ to generate the next feasible point $\left(w^{(\kappa+1)}, \theta^{(\kappa+1)}\right)$ :

$$
\begin{aligned}
\max _{\mathbf{w}, \boldsymbol{\theta}} \frac{1}{K} \sum_{k=1}^{K} \frac{r_{k}(\mathbf{w}, \boldsymbol{\theta})}{r_{k}\left(w^{(\kappa)}, \theta^{(\kappa)}\right)} f\left(r_{1}\left(w^{(\kappa)}, \theta^{(\kappa)}\right)\right. \\
\left., \ldots, r_{K}\left(w^{(\kappa)}, \theta^{(\kappa)}\right)\right) \text { s.t. } \quad(7 b),
\end{aligned}
$$

which is equivalent to the following problem:

$$
\max _{\mathbf{w}, \boldsymbol{\theta}} f^{(\kappa)}(\mathbf{w}, \boldsymbol{\theta}) \triangleq \sum_{k=1}^{K} \gamma_{k}^{(\kappa)} r_{k}(\mathbf{w}, \boldsymbol{\theta}) \quad \text { s.t. } \quad(7 b),
$$

for

$\gamma_{k}^{(\kappa)} \triangleq \frac{f\left(r_{1}\left(w^{(\kappa)}, \theta^{(\kappa)}\right), \ldots, r_{K}\left(w^{(\kappa)}, \theta^{(\kappa)}\right)\right)}{r_{k}\left(w^{(\kappa)}, \theta^{(\kappa)}\right)}, k=1, \ldots, K$.

\section{A. Beamforming descent iteration}

To generate $w^{(\kappa+1)}$ we seek $w^{(\kappa+1)}$, so that the following holds:

$$
f^{(\kappa)}\left(w^{(\kappa+1)}, \theta^{(\kappa)}\right)>f^{(\kappa)}\left(w^{(\kappa)}, \theta^{(\kappa)}\right) .
$$

Using the inequality [1]

$$
\ln \left|I_{n}+[\mathbf{V}]^{2}(\mathbf{Y})^{-1}\right| \geq \ln \left|I_{n}+[\bar{V}]^{2}(\bar{Y})^{-1}\right|
$$




$$
\begin{array}{r}
-\left\langle[\bar{V}]^{2}(\bar{Y})^{-1}\right\rangle+2 \Re\left\{\left\langle\bar{V}^{H}(\bar{Y})^{-1} \mathbf{V}\right\rangle\right\} \\
-\left\langle(\bar{Y})^{-1}-\left(\bar{Y}+[\bar{V}]^{2}\right)^{-1},[\mathbf{V}]^{2}+\mathbf{Y}\right\rangle, \\
\forall \mathbf{V}, \mathbf{Y} \succ 0 \quad \& \quad \bar{V}, \bar{Y} \succ 0,
\end{array}
$$

for $\mathbf{V}=\mathcal{H}_{k}\left(\theta^{(\kappa)}\right) \mathbf{w}_{k}, \mathbf{Y}=\sum_{j \in \mathcal{K} \backslash\{k\}}\left|\mathcal{H}_{k}\left(\theta^{(\kappa)}\right) \mathbf{w}_{j}\right|^{2}+\sigma$, and $\bar{V}=\mathcal{H}_{k}\left(\theta^{(\kappa)}\right) w_{k}^{(\kappa)}, \bar{Y}=y_{k}^{(\kappa)} \triangleq$ $\sum_{j \in \mathcal{K} \backslash\{k\}}\left|\mathcal{H}_{k}\left(\theta^{(\kappa)}\right) w_{j}^{(\kappa)}\right|^{2}+\sigma$, yields

$$
\begin{aligned}
r_{k}\left(\mathbf{w}, \theta^{(\kappa)}\right) \geq & r_{k}^{(\kappa)}(\mathbf{w}) \\
\triangleq & a_{k}^{(\kappa)}+2 \Re\left\{\left\langle b_{k}^{(\kappa)}, \mathbf{w}_{k}\right\rangle\right\} \\
& -c_{k}^{(\kappa)} \sum_{j=1}^{K}\left|\mathcal{H}_{k}\left(\theta^{(\kappa)}\right) \mathbf{w}_{j}\right|^{2},
\end{aligned}
$$

with $a_{k}^{(\kappa)} \triangleq r_{k}\left(w^{(\kappa)}, \theta^{(\kappa)}\right)-\left|\mathcal{H}_{k}\left(\theta^{(\kappa)}\right) w_{k}^{(\kappa)}\right|^{2} / y_{k}^{(\kappa)}-\sigma c_{k}^{(\kappa)}$, $b_{k}^{(\kappa)} \triangleq \mathcal{H}_{k}^{H}\left(\theta^{(\kappa)}\right) \mathcal{H}_{k}\left(\theta^{(\kappa)}\right) w_{k}^{(\kappa)} / y_{k}^{(\kappa)}$, and $0<c_{k}^{(\kappa)} \triangleq$ $\left|\mathcal{H}_{k}\left(\theta^{(\kappa)}\right) w_{k}^{(\kappa)}\right|^{2} /\left[y_{k}^{(\kappa)}\left(y_{k}^{(\kappa)}+\left|\mathcal{H}_{k}\left(\theta^{(\kappa)}\right) w_{k}^{(\kappa)}\right|^{2}\right)\right]$.

The function $r_{k}^{(\kappa)}(\mathbf{w})$ is seen to be concave quadratic, which matches with $r_{k}^{(\kappa)}\left(\mathbf{w}, \theta^{(\kappa)}\right)$ at $w^{(\kappa)}$. We solve the following convex problem at the $\kappa$-th iteration to generate $w^{(\kappa+1)}$ :

$$
\max _{\mathbf{w}} f_{b}^{(\kappa)}(\mathbf{w}) \quad \text { s.t. } \quad(7 b),
$$

where

$$
\begin{aligned}
f_{b}^{(\kappa)}(\mathbf{w}) \triangleq & \sum_{k=1}^{K} \gamma_{k}^{(\kappa)} r_{k}^{(\kappa)}(\mathbf{w}) \\
= & \sum_{k=1}^{K} \gamma_{k}^{(\kappa)} a_{k}^{(\kappa)}+2 \sum_{k=1}^{K} \Re\left\{\left\langle\gamma_{k}^{(\kappa)} b_{k}^{(\kappa)}, \mathbf{w}_{k}\right\rangle\right\} \\
& -\sum_{k=1}^{K}\left(\mathbf{w}_{k}\right)^{H} \Psi^{(\kappa)} \mathbf{w}_{k}
\end{aligned}
$$

with $0 \preceq \Psi^{(\kappa)} \triangleq \sum_{j=1}^{K} \gamma_{j}^{(\kappa)} c_{j}^{(\kappa)} \mathcal{H}_{j}^{H}\left(\theta^{(\kappa)}\right) \mathcal{H}_{j}\left(\theta^{(\kappa)}\right)$. By using the Lagrangian multiplier method, we obtain the following closed-form solution of $(16)^{1}$

$w_{k}^{(\kappa+1)}=\left\{\begin{array}{l}\left(\Psi^{(\kappa)}\right)^{-1} \gamma_{k}^{(\kappa)} b_{k}^{(\kappa)} \text { if } \sum_{k=1}^{K}\left\|\left(\Psi^{(\kappa)}\right)^{-1} \gamma_{k}^{(\kappa)} b_{k}^{(\kappa)}\right\|^{2} \leq P \\ \left(\Psi^{(\kappa)}+\mu I_{M}\right)^{-1} \gamma_{k}^{(\kappa)} b_{k}^{(\kappa)} \quad \text { otherwise, }\end{array}\right.$

where $\mu>0$ is chosen by bisection such that $\sum_{k=1}^{K}\left\|\left(\Psi^{(\kappa)}+\mu I_{M}\right)^{-1} \gamma_{k}^{(\kappa)} b_{k}^{(\kappa)}\right\|^{2}=P$.

\section{B. Programmable reflecting elements' descent iteration}

We seek the next iterative point $\theta^{(\kappa+1)}$ such that

$$
f^{(\kappa)}\left(w^{(\kappa+1)}, \theta^{(\kappa+1)}\right)>f^{(\kappa)}\left(w^{(\kappa+1)}, \theta^{(\kappa)}\right) .
$$

Using the inequality (14) for $\mathbf{V}=\mathcal{H}_{k}(\boldsymbol{\theta}) w_{k}^{(\kappa+1)}, \mathbf{Y}=$ $\sum_{j \in \mathcal{K} \backslash\{k\}}\left|\mathcal{H}_{k}(\boldsymbol{\theta}) w_{j}^{(\kappa+1)}\right|^{2}+\sigma$, and $\bar{V}=\mathcal{H}_{k}\left(\theta^{(\kappa)}\right) w_{k}^{(\kappa+1)}$, $\bar{Y}=y_{k}^{(\kappa+1)} \triangleq \sum_{j \in \mathcal{K} \backslash\{k\}}\left|\mathcal{H}_{k}\left(\theta^{(\kappa)}\right) w_{j}^{(\kappa+1)}\right|^{2}+\sigma$, yields

$$
r_{k}\left(w^{(\kappa+1)}, \boldsymbol{\theta}\right) \geq \tilde{r}_{k}^{(\kappa)}(\boldsymbol{\theta})
$$

${ }^{1}\left(\Psi^{(\kappa)}\right)^{-1}$ is understood as the pseudo-inversion when $\Psi^{(\kappa)} \succeq 0$

$$
\begin{aligned}
\triangleq & \frac{2 \Re\left\{\left(w_{k}^{(\kappa+1)}\right)^{H} \mathcal{H}_{k}^{H}\left(\theta^{(\kappa)}\right) \mathcal{H}_{k}(\boldsymbol{\theta}) w_{k}^{(\kappa+1)}\right\}}{y_{k}^{(\kappa+1)}} \\
& +\tilde{a}_{k}^{(\kappa)}-\tilde{\tilde{c}}_{k}^{(\kappa)} \sum_{j=1}^{K}\left|\mathcal{H}_{k}(\boldsymbol{\theta}) w_{j}^{(\kappa+1)}\right|^{2}, \quad(20)
\end{aligned}
$$

with $\tilde{a}_{k}^{(\kappa)} \triangleq r_{k}\left(w^{(\kappa+1)}, \theta^{(\kappa)}\right)-\left|\mathcal{H}_{k}\left(\theta^{(\kappa)}\right) w_{k}^{(\kappa+1)}\right|^{2} / y_{k}^{(\kappa+1)}-$ $\sigma \tilde{\tilde{c}}_{k}^{(\kappa)}$ and $0<\tilde{\tilde{c}}_{k}^{(\kappa)} \triangleq\left|\mathcal{H}_{k}\left(\theta^{(\kappa)}\right) w_{k}^{(\kappa+1)}\right|^{2} /\left[y_{k}^{(\kappa+1)}\left(y_{k}^{(\kappa+1)}\right.\right.$ $\left.\left.+\left|\mathcal{H}_{k}\left(\theta^{(\kappa)}\right) w_{k}^{(\kappa+1)}\right|^{2}\right)\right]$.

Let us define $\Upsilon_{n}$ as the matrix of size $N \times N$ having only zero entries, except for its $(n, n)$-entry, which is 1 , to express

$$
\operatorname{diag}\left(e^{\jmath \boldsymbol{\theta}}\right)=\sum_{n=1}^{N} e^{\jmath \boldsymbol{\theta}_{n}} \Upsilon_{n}
$$

We then use (4) to arrive at:

$$
\begin{array}{r}
\left(w_{k}^{(\kappa+1)}\right)^{H} \mathcal{H}_{k}^{H}\left(\theta^{(\kappa)}\right) \mathcal{H}_{k}(\boldsymbol{\theta}) w_{k}^{(\kappa+1)}= \\
\left(w_{k}^{(\kappa+1)}\right)^{H} \mathcal{H}_{k}^{H}\left(\theta^{(\kappa)}\right)\left[\tilde{h}_{\mathrm{BR}-\mathrm{k}} \operatorname{diag}\left(e^{\jmath \boldsymbol{\theta}}\right) H_{\mathrm{B}-\mathrm{R}}+\tilde{h}_{\mathrm{B}-\mathrm{k}}\right] w_{k}^{(\kappa+1)}= \\
\left(w_{k}^{(\kappa+1)}\right)^{H} \mathcal{H}_{k}^{H}\left(\theta^{(\kappa)}\right) \tilde{h}_{\mathrm{B}-\mathrm{k}} w_{k}^{(\kappa+1)} \\
+\left(w_{k}^{(\kappa+1)}\right)^{H} \mathcal{H}_{k}^{H}\left(\theta^{(\kappa)}\right) \tilde{h}_{\mathrm{BR}-\mathrm{k}} \operatorname{diag}\left(e^{\jmath \boldsymbol{\theta}}\right) H_{\mathrm{B}-\mathrm{R}} w_{k}^{(\kappa+1)}= \\
\left(w_{k}^{(\kappa+1)}\right)^{H} \mathcal{H}_{k}^{H}\left(\theta^{(\kappa)}\right) \tilde{h}_{\mathrm{B}-\mathrm{k}} w_{k}^{(\kappa+1)} \\
+\sum_{n=1}^{N}\left(w_{k}^{(\kappa+1)}\right)^{H} \mathcal{H}_{k}^{H}\left(\theta^{(\kappa)}\right) \tilde{h}_{\mathrm{BR}-\mathrm{k}} \Upsilon_{n} H_{\mathrm{B}-\mathrm{R}} w_{k}^{(\kappa+1)} e^{\jmath \boldsymbol{\theta}_{n}}= \\
\alpha_{k}^{(\kappa)}+\sum_{n=1}^{N} \tilde{b}_{k}^{(\kappa)}(n) e^{\jmath \theta_{n}},
\end{array}
$$

with $\alpha_{k}^{(\kappa)} \triangleq\left(w_{k}^{(\kappa+1)}\right)^{H} \mathcal{H}_{k}^{H}\left(\theta^{(\kappa)}\right) \tilde{h}_{\mathrm{B}-\mathrm{k}} w_{k}^{(\kappa+1)}$, and ${ }^{2} \tilde{b}_{k}^{(\kappa)}(n)=$ $\left(w_{k}^{(\kappa+1)}\right)^{H} \mathcal{H}_{k}^{H}\left(\theta^{(\kappa)}\right) \tilde{h}_{\mathrm{BR}-\mathrm{k}} \Upsilon_{n} H_{\mathrm{B}-\mathrm{R}} w_{k}^{(\kappa+1)}, n=1, \ldots N$.

To expound further, we have:

$$
\begin{aligned}
\left|\mathcal{H}_{k}(\boldsymbol{\theta}) w_{j}^{(\kappa+1)}\right|^{2}= & \left|\left(\tilde{h}_{\mathrm{BR}-\mathrm{k}} \operatorname{diag}\left(e^{\jmath \boldsymbol{\theta}}\right) H_{\mathrm{B}-\mathrm{R}}+\tilde{h}_{\mathrm{B}-\mathrm{k}}\right) w_{j}^{(\kappa+1)}\right|^{2} \\
= & \left|\tilde{h}_{\mathrm{BR}-\mathrm{k}} \operatorname{diag}\left(e^{\jmath \boldsymbol{\theta}}\right) H_{\mathrm{B}-\mathrm{R}} w_{j}^{(\kappa+1)}\right|^{2} \\
& +2 \Re\left\{\left(w_{j}^{(\kappa+1)}\right)^{H}\left(\tilde{h}_{\mathrm{B}-\mathrm{k}}\right)^{H} \tilde{h}_{\mathrm{BR}-\mathrm{k}}\right. \\
& \left.\operatorname{diag}\left(e^{\jmath \boldsymbol{\theta}}\right) H_{\mathrm{B}-\mathrm{R}} w_{j}^{(\kappa+1)}\right\}+\left|\tilde{h}_{\mathrm{B}-\mathrm{k}} w_{j}^{(\kappa+1)}\right|^{2} \\
= & \left|\tilde{h}_{\mathrm{BR}-\mathrm{k}} \operatorname{diag}\left(e^{\jmath \boldsymbol{\theta}}\right) H_{\mathrm{B}-\mathrm{R}} w_{j}^{(\kappa+1)}\right|^{2} \\
& +2 \Re\left\{\sum_{n=1}^{N}\left(w_{j}^{(\kappa+1)}\right)^{H}\left(\tilde{h}_{\mathrm{B}-\mathrm{k}}\right)^{H} \tilde{h}_{\mathrm{BR}-\mathrm{k}} \Upsilon_{n}\right. \\
& \left.H_{\mathrm{B}-\mathrm{R}} w_{j}^{(\kappa+1)} e^{\jmath \boldsymbol{\theta}}\right\}+\left|\tilde{h}_{\mathrm{B}-\mathrm{k}} w_{j}^{(\kappa+1)}\right|^{2} .(22)
\end{aligned}
$$

Furthermore,

$$
\begin{aligned}
& \tilde{h}_{\mathrm{BR}-\mathrm{k}} \operatorname{diag}\left(e^{\boldsymbol{\theta}}\right) H_{\mathrm{B}-\mathrm{R}} w_{j}^{(\kappa+1)} \\
= & \tilde{h}_{\mathrm{BR}-\mathrm{k}}\left(\sum_{n=1}^{N} e^{\boldsymbol{\theta}_{n}} \Upsilon_{n}\right) H_{\mathrm{B}-\mathrm{R}} w_{j}^{(\kappa+1)} \\
= & \sum_{n=1}^{N} \alpha_{k, j}^{(\kappa+1)}(n) e^{\jmath \theta_{n}},
\end{aligned}
$$

\footnotetext{
${ }^{2}$ In what follows $b(i)$ is the $i$-th entry of $b$ and $[A](i, i)$ is the $i$-th diagonal entry of $A$, and $[A]^{*}(i, i)$ is the complex conjugate of $[A](i, i)$
} 
for $\alpha_{k, j}^{(\kappa+1)}(n)=\tilde{h}_{\mathrm{BR}-\mathrm{k}} \Upsilon_{n} H_{\mathrm{B}-\mathrm{R}} w_{j}^{(\kappa+1)}, n=1, \ldots, N$.

Based on (20), (21), (22), and (23), we obtain

$$
\begin{aligned}
\tilde{r}_{k}^{(\kappa)}(\boldsymbol{\theta})= & \tilde{a}_{k}^{(\kappa+1)}+2 \Re\left\{\sum_{n=1}^{N} \tilde{b}_{k}^{(\kappa)}(n) e^{\jmath \theta_{n}}\right\} \\
& -\tilde{\tilde{c}}_{k}^{(\kappa)} \sum_{j=1}^{K}\left|\sum_{n=1}^{N} \alpha_{k, j}^{(\kappa+1)}(n) e^{\jmath \boldsymbol{\theta}_{n}}\right|^{2} \\
= & \tilde{a}_{k}^{(\kappa+1)}+2 \Re\left\{\sum_{n=1}^{N} \tilde{b}_{k}^{(\kappa)}(n) e^{\jmath \theta_{n}}\right\} \\
& -\tilde{\tilde{c}}_{k}^{(\kappa)} \sum_{j=1}^{K}\left(e^{\jmath \boldsymbol{\theta}}\right)^{H} \Phi_{k, j}^{(\kappa+1)} e^{\jmath \boldsymbol{\theta}},
\end{aligned}
$$

where $\tilde{a}_{k}^{(\kappa+1)} \triangleq \tilde{a}_{k}^{(\kappa)}+2 \Re\left\{\alpha_{k}^{(\kappa)}\right\} / y_{k}^{(\kappa+1)}-$ $\tilde{\tilde{c}}_{k}^{(\kappa)} \sum_{j=1}^{N}\left|\tilde{h}_{\mathrm{B}-\mathrm{k}} w_{j}^{(\kappa+1)}\right|^{2}, \quad \tilde{b}_{k}^{(\kappa+1)}(n) \triangleq \tilde{b}_{k}^{(\kappa)}(n) / y_{k}^{(\kappa+1)}-$ $\tilde{\tilde{c}}_{k}^{(\kappa)} \sum_{j=1}^{K}\left(w_{j}^{(\kappa+1)}\right)^{H}\left(\tilde{h}_{\mathrm{B}-\mathrm{k}}\right)^{H} \tilde{h}_{\mathrm{BR}-\mathrm{k}} \Upsilon_{n} H_{\mathrm{B}-\mathrm{R}} w_{j}^{(\kappa+1)}, \quad$ and $\Phi_{k, j}^{(\kappa+1)}(n, m)=\left(\alpha_{k, j}^{(\kappa+1)}(n)\right)^{*} \alpha_{k, j}^{(\kappa+1)}(m), n=1, \ldots, N ; m=$ $1, \ldots, N$.

Note that $\Phi_{k, j}^{(\kappa+1)} \succeq 0$. Therefore,

$$
\begin{aligned}
f_{c}^{(\kappa)}(\boldsymbol{\theta}) \triangleq & \sum_{k=1}^{K} \gamma_{k}^{(\kappa)} \tilde{r}_{k}^{(\kappa)}(\boldsymbol{\theta}) \\
= & \tilde{a}^{(\kappa+1)}+2 \Re\left\{\sum_{n=1}^{N} \tilde{b}^{(\kappa+1)}(n) e^{\jmath \theta_{n}}\right\} \\
& -\left(e^{\jmath \boldsymbol{\theta}}\right)^{H} \Phi^{(\kappa+1)} e^{\jmath \boldsymbol{\theta}},
\end{aligned}
$$

for $\quad \tilde{a}^{(\kappa+1)} \triangleq \sum_{k=1}^{K} \gamma_{k}^{(\kappa)} \tilde{a}_{k}^{(\kappa+1)}, \quad \tilde{b}^{(\kappa+1)}(n)$

$\sum_{k=1}^{K} \gamma_{k}^{(\kappa)} \tilde{b}_{k}^{(\kappa+1)}(n), n=1, \ldots, N$, and $0 \preceq \Phi^{(\kappa+1)} \triangleq$ $\sum_{k=1}^{K} \sum_{j=1}^{N} \gamma_{k}^{(\kappa)} \tilde{\tilde{c}}_{k}^{(\kappa)} \Phi_{k, j}^{(\kappa+1)}$.

We use the following problem at the $\kappa$-th iteration to generate $\theta^{(\kappa+1)}$ :

$$
\max _{\boldsymbol{\theta}} f_{c}^{(\kappa)}(\boldsymbol{\theta}) .
$$

Following [36], we have (27). We thus solve the following problem at the $\kappa$-th iteration to generate $\theta^{(\kappa+1)}$ :

$$
\max _{\boldsymbol{\theta}} \tilde{f}_{c}^{(\kappa)}(\boldsymbol{\theta})
$$

where the function $\tilde{f}_{c}^{(\kappa)}(\boldsymbol{\theta})$ is an affine function of $e^{\jmath \boldsymbol{\theta}}$. By noting that $\Re\left\{c e^{\jmath \theta_{n}}\right\}=|c| \cos \left(\angle c+\theta_{n}\right)$ and thus it is maximized at $\theta_{n}=-\angle c$, we obtain the closed-form solution of (28) $\mathrm{as}^{3}$

$$
\begin{aligned}
\theta_{n}^{(\kappa+1)}= & -\angle\left(\tilde{b}^{(\kappa+1)}(n)-\sum_{m=1}^{N} e^{-\jmath \theta_{m}^{(\kappa)}} \Phi^{(\kappa+1)}(m, n)\right. \\
& \left.+\lambda_{\max }\left(\Phi^{(\kappa+1)}\right) e^{-\jmath \theta_{n}^{(\kappa)}}\right), n=1, \ldots, N .
\end{aligned}
$$

It follows from (27) that $f^{(\kappa)}\left(w^{(\kappa+1)}, \theta^{(\kappa+1)}\right) \geq$ $f_{c}^{(\kappa)}\left(\theta^{(\kappa+1)}\right) \geq \tilde{f}_{c}^{(\kappa)}\left(\theta^{(\kappa+1)}\right)>\tilde{f}_{c}^{(\kappa)}\left(\theta^{(\kappa)}\right)=f_{c}^{(\kappa)}\left(\theta^{(\kappa)}\right)=$ $f^{(\kappa)}\left(w^{(\kappa+1)}, \theta^{(\kappa)}\right)$, confirming (19), so $\theta^{(\kappa+1)}$ is a better feasible point than $\theta^{(\kappa)}$.

$$
{ }^{3}\left[\left(\Phi^{(\kappa+1)}-\mu I_{N}\right) e^{\jmath \theta^{(\kappa)}}\right](n) \text { is the } n \text {-th entry of }\left(\Phi^{(\kappa+1)}-\mu I_{N}\right) e^{\jmath \theta^{(\kappa)}}
$$

\section{Proper Gaussian Signaling Geometric Mean Rate Opti- mization}

Algorithm 1 provides the pseudo-code for the proposed computational procedure of steepest descent for computing (9) as the iterations (18) and (29) seek a descent direction by seeking a better feasible point for the nonconvex problem (10) instead of seeking its optimal solution for reducing the computational load with guaranteed convergence, as it is often done in the context of the Frank-and-Wolfe method [37]. Of course, one can still seek the optimal solution of (10) for the steepest descent by iterating (18) and (29) many times, because according to [27], this kind of alternating descent iterations often converge to at least a locally optimal solution of (10). The global optimality can not be proved theoretically, but we found that it is globally optimal in many cases.

To the best of our knowledge, there is no the conventional descent algorithm, because the conception of descent descent algorithms is a research branch in computational optimization and what make descent algorithms different is the specific way they choose their a descent directions. Hence, our descent directions are completely new and rather different from the popular steepest descent techniques. Furthermore, all other exiting algorithms, which solve convex problems and iteratively at a high complexity are very sensitive to the problem sizes. However, our algorithms iterate using closed- form expressions, hence their complexity is low.

\begin{tabular}{l}
\hline Algorithm 1 PGS GM descent algorithm \\
\hline 1: Initialization: Set $\kappa=0$. Randomly generate $\left(w^{(0)}, \theta^{(0)}\right)$ \\
satisfying the constraint (7b) and define $\gamma^{(0)}$ by $(12)$. \\
2: Repeat until convergence of the objective in (8): Gener- \\
ate $w^{(\kappa+1)}$ by $(18)$ and $\theta^{(\kappa+1)}$ by $(29)$. Reset $\kappa \leftarrow \kappa+1$. \\
3: Output $\left(w^{(\kappa)}, \theta^{(\kappa)}\right)$ and rates $r_{k}\left(w^{(\kappa)}, \theta^{(\kappa)}\right), k=$ \\
$1, \ldots, K$ with their GM $\left(\prod_{k=1}^{K} r_{k}\left(w^{(\kappa)}, \theta^{(\kappa)}\right)\right)^{1 / K}$. \\
\hline
\end{tabular}

\section{IMPROPER GAUSSIAN SIGNALING}

In (1), the proper Gaussian sources $s_{k}$ are linearly beamformed by the beamformers $\mathbf{w}_{k}$, hence the transmit signal $x$ is also proper Gaussian, i.e. $\mathbb{E}\left(x x^{T}\right)=$ $\sum_{k \in \mathcal{K}} \mathbf{w}_{k}\left(\mathbf{w}_{k}\right)^{T} \mathbb{E}\left[\left(s_{k}\right)^{2}\right]=0$. In this section, the proper Gaussian sources $s_{k}$ are widely linearly beamformed by the pairs of beamformers $\mathbf{w}_{1, k} \in \mathbb{C}^{M}$ and $\mathbf{w}_{2, k} \in \mathbb{C}^{M}$ as in [38]

$$
\left[\begin{array}{ll}
\mathbf{w}_{1, k} & \mathbf{w}_{2, k}
\end{array}\right]\left[\begin{array}{l}
s_{k} \\
s_{k}^{*}
\end{array}\right],
$$

resulting in the transmit signal

$$
x=\sum_{k=1}^{K}\left(\mathbf{w}_{1, k} s_{k}+\mathbf{w}_{2, k} s_{k}^{*}\right),
$$

and for improper Gaussian, as

$$
\mathbb{E}\left(x x^{T}\right)=\sum_{k=1}^{K}\left(\mathbf{w}_{1, k} \mathbf{w}_{2, k}^{T}+\mathbf{w}_{2, k} \mathbf{w}_{1, k}^{T}\right) \mathbb{E}\left(\left|s_{k}\right|^{2}\right) \neq 0 .
$$




$$
\begin{aligned}
f_{c}^{(\kappa)}(\boldsymbol{\theta})= & \tilde{a}^{(\kappa+1)}+2 \Re\left\{\sum_{n=1}^{N} \tilde{b}^{(\kappa+1)}(n) e^{\jmath \theta_{n}}\right\}-\left(e^{\jmath \boldsymbol{\theta}}\right)^{H}\left(\Phi^{(\kappa+1)}-\lambda_{\max }\left(\Phi^{(\kappa+1)}\right) I_{N}\right) e^{\jmath \boldsymbol{\theta}}-\lambda_{\max }\left(\Phi^{(\kappa+1)}\right)\left(e^{\jmath \boldsymbol{\theta}}\right)^{H} I_{N} e^{\jmath \boldsymbol{\theta}} \\
= & \tilde{a}^{(\kappa+1)}+2 \Re\left\{\sum_{n=1}^{N} \tilde{b}^{(\kappa+1)}(n) e^{\jmath \theta_{n}}\right\}-\left(e^{\jmath \boldsymbol{\theta}}\right)^{H}\left(\Phi^{(\kappa+1)}-\lambda_{\max }\left(\Phi^{(\kappa+1)}\right) I_{N}\right) e^{\jmath \boldsymbol{\theta}}-\lambda_{\max }\left(\Phi^{(\kappa+1)}\right) N \\
\geq & \tilde{f}_{c}^{(\kappa)}(\boldsymbol{\theta}) \\
\triangleq & \tilde{a}^{(\kappa+1)}+2 \Re\left\{\sum_{n=1}^{N} \tilde{b}^{(\kappa+1)}(n) e^{\jmath \theta_{n}}\right\}-\left[2 \Re\left\{\left(e^{\jmath \theta^{(\kappa)}}\right)^{H}\left(\Phi^{(\kappa+1)}-\lambda_{\max }\left(\Phi^{(\kappa+1)}\right) I_{N}\right) e^{\jmath \boldsymbol{\theta}}\right\}\right. \\
& \left.-\left(e^{\jmath \theta^{(\kappa)}}\right)^{H}\left(\Phi^{(\kappa+1)}-\lambda_{\max }\left(\Phi^{(\kappa+1)}\right) I_{N}\right) e^{\jmath^{(\kappa)}}\right]-\lambda_{\max }\left(\Phi^{(\kappa+1)}\right) N \\
= & \tilde{a}^{(\kappa+1)}+2 \Re\left\{\sum_{n=1}^{N}\left(\tilde{b}^{(\kappa+1)}(n)-\sum_{m=1}^{N} e^{-\jmath \theta_{m}^{(\kappa)}} \Phi^{(\kappa+1)}(m, n)+\lambda_{\max }\left(\Phi^{(\kappa+1)}\right) e^{-\jmath \theta_{n}^{(\kappa)}}\right) e^{\jmath \theta_{n}}\right\} \\
& -\left(e^{\jmath \theta^{(\kappa)}}\right)^{H} \Phi^{(\kappa+1)} e^{\jmath \theta^{(\kappa)}}-2 \lambda_{\max }\left(\Phi^{(\kappa+1)}\right) N .
\end{aligned}
$$

The equation (2) of the received signal at UE $k$ becomes:

$$
y_{k}=\mathcal{H}_{k}(\boldsymbol{\theta}) \sum_{k=1}^{K}\left(\mathbf{w}_{1, k} s_{k}+\mathbf{w}_{2, k} s_{k}^{*}\right)+n_{k} .
$$

We augment (32) as

$$
\begin{aligned}
{\left[\begin{array}{l}
y_{k} \\
y_{k}^{*}
\end{array}\right] } & =\left[\begin{array}{cc}
\mathcal{H}_{k}(\boldsymbol{\theta}) & 0 \\
0 & \mathcal{H}_{k}^{*}(\boldsymbol{\theta})
\end{array}\right] \sum_{k=1}^{K}\left[\begin{array}{ll}
\mathbf{w}_{1, k} & \mathbf{w}_{2, k} \\
\mathbf{w}_{2, k}^{*} & \mathbf{w}_{1, k}^{*}
\end{array}\right]\left[\begin{array}{l}
s_{k} \\
s_{k}^{*}
\end{array}\right]+\left[\begin{array}{l}
n_{k} \\
n_{k}^{*}
\end{array}\right] \\
& =\Lambda_{k}(\boldsymbol{\theta}) \sum_{k=1}^{K} \mathbf{W}_{k} \bar{s}_{k}+\bar{n}_{k}
\end{aligned}
$$

for the linear mappings $\Lambda_{k}(\boldsymbol{\theta}) \triangleq\left[\begin{array}{cc}\mathcal{H}_{k}(\boldsymbol{\theta}) & 0 \\ 0 & \mathcal{H}_{k}^{*}(\boldsymbol{\theta})\end{array}\right] \in$ $\mathbb{C}^{2 \times(2 M)}$, and $\mathbf{W}_{k} \triangleq\left[\begin{array}{ll}\mathbf{w}_{1, k} & \mathbf{w}_{2, k} \\ \mathbf{w}_{2, k}^{*} & \mathbf{w}_{1, k}^{*}\end{array}\right] \in \mathbb{C}^{2 M \times 2}$, and $\bar{s}_{k} \triangleq$ $\left[\begin{array}{l}s_{k} \\ s_{k}^{*}\end{array}\right] \in \mathbb{C}^{2}, \bar{n}_{k} \triangleq\left[\begin{array}{l}n_{k} \\ n_{k}^{*}\end{array}\right] \in \mathbb{C}^{2}$.

For $\mathbf{w} \triangleq\left\{\mathbf{w}_{k} \triangleq\left[\begin{array}{l}\mathbf{w}_{1, k} \\ \mathbf{w}_{2, k}\end{array}\right] \in \mathbb{C}^{2 M}: k \in \mathcal{K}\right\}$, the rate at $\mathrm{UE}$ $k$ is calculated by $(1 / 2) r_{k}(\mathbf{w}, \boldsymbol{\theta})$ [39] with

$$
\begin{aligned}
& r_{k}(\mathbf{w}, \boldsymbol{\theta})= \\
& \ln \left|I_{2}+\left[\Lambda_{k}(\boldsymbol{\theta}) \mathbf{W}_{k}\right]^{2}\left(\sum_{j \in \mathcal{K} \backslash\{k\}}\left[\Lambda_{k}(\boldsymbol{\theta}) \mathbf{W}_{j}\right]^{2}+\sigma I_{2}\right)^{-1}\right| .
\end{aligned}
$$

For the particular class of $\mathbf{w}_{2, k} \equiv 0$, i.e. when $x$ in (31) is proper Gaussian, it may be shown that

$$
\begin{aligned}
& r_{k}(\mathbf{w}, \boldsymbol{\theta})= \\
& \quad 2 \ln \left(1+\left|\mathcal{H}_{k}(\boldsymbol{\theta}) \mathbf{w}_{1, k}\right|^{2} /\left(\sum_{j \in \mathcal{K} \backslash\{k\}}\left|\mathcal{H}_{k}(\boldsymbol{\theta}) \mathbf{w}_{1, j}\right|^{2}+\sigma\right)\right),
\end{aligned}
$$

hence $(1 / 2) r_{k}(\mathbf{w}, \boldsymbol{\theta})$ is the known rate (6).

Like (8), the problem of maximizing the GM for users' rates corresponding IGS is thus formulated as

$$
\min _{\mathbf{w}, \boldsymbol{\theta}} f\left(r_{1}(\mathbf{w}, \boldsymbol{\theta}), \ldots, r_{K}(\mathbf{w}, \boldsymbol{\theta})\right) \triangleq \frac{1}{\left(\prod_{k=1}^{K} r_{k}(\mathbf{w}, \boldsymbol{\theta})\right)^{1 / K}}(35
$$

$$
\text { s.t. } \sum_{k=1}^{K}\left(\left\|\mathbf{w}_{1, k}\right\|^{2}+\left\|\mathbf{w}_{2, k}\right\|^{2}\right) \leq P .
$$

Let $\left(w^{(\kappa)}, \theta^{(\kappa)}\right)$ be a feasible point for (35) that is found from the $(\kappa-1)$-st round. Like (11), we use the following steepest descent optimization for the convex function $f\left(r_{1}, \ldots, r_{K}\right)=1 /\left(\prod_{k=1}^{K} r_{k}\right)^{1 / K}$ to generate the next feasible point $\left(w^{(\kappa+1)}, \theta^{(\kappa+1)}\right)$ :

$$
\max _{\mathbf{w}, \boldsymbol{\theta}} F^{(\kappa)}(\mathbf{w}, \boldsymbol{\theta}) \triangleq \sum_{k=1}^{K} \gamma_{k}^{(\kappa)} r_{k}(\mathbf{w}, \boldsymbol{\theta}) \quad \text { s.t. }
$$

where

$\gamma_{k}^{(\kappa)} \triangleq \frac{f\left(r_{1}\left(w^{(\kappa)}, \theta^{(\kappa)}\right), \ldots, r_{K}\left(w^{(\kappa)}, \theta^{(\kappa)}\right)\right)}{r_{k}\left(w^{(\kappa)}, \theta^{(\kappa)}\right)}, k=1, \ldots, K$.

Another way of defining the UEs' rates is through the equivalent composite real system for (32):

$$
\begin{aligned}
& \tilde{y}_{k} \triangleq\left[\begin{array}{c}
\Re\left\{y_{k}\right\} \\
\Im\left\{y_{k}\right\}
\end{array}\right]= \\
& {\left[\begin{array}{cc}
\Re\left\{\mathcal{H}_{k}(\boldsymbol{\theta})\right\} & -\Im\left\{\mathcal{H}_{k}(\boldsymbol{\theta})\right\} \\
\Im\left\{\mathcal{H}_{k}(\boldsymbol{\theta})\right\} & \Re\left\{\mathcal{H}_{k}(\boldsymbol{\theta})\right\}
\end{array}\right]} \\
& \sum_{j=1}^{K}\left[\begin{array}{lc}
\Re\left\{\mathbf{w}_{1, j}\right\}+\Re\left\{\mathbf{w}_{2, j}\right\} & -\Im\left\{\mathbf{w}_{1, j}\right\}+\Im\left\{\mathbf{w}_{2, j}\right\} \\
\Im\left\{\mathbf{w}_{1, j}\right\}+\Im\left\{\mathbf{w}_{2, j}\right\} & \Re\left\{\mathbf{w}_{1, j}\right\}-\Re\left\{\mathbf{w}_{2, j}\right\}
\end{array}\right] \\
& \times\left[\begin{array}{c}
\Re\left\{s_{j}\right\} \\
\Im\left\{s_{j}\right\}
\end{array}\right]+\left[\begin{array}{c}
\Re\left\{n_{k}\right\} \\
\Im\left\{n_{k}\right\}
\end{array}\right]= \\
& \overline{\mathcal{H}}_{k}(\boldsymbol{\theta}) \sum_{j=1}^{K} \mathbf{V}_{j} \tilde{s}_{j}+\tilde{n}_{k},
\end{aligned}
$$

where we have:

$$
\begin{array}{r}
\overline{\mathcal{H}}_{k}(\boldsymbol{\theta}) \triangleq\left[\begin{array}{cc}
\Re\left\{\mathcal{H}_{k}(\boldsymbol{\theta})\right\} & -\Im\left\{\mathcal{H}_{k}(\boldsymbol{\theta})\right\} \\
\Im\left\{\mathcal{H}_{k}(\boldsymbol{\theta})\right\} & \Re\left\{\mathcal{H}_{k}(\boldsymbol{\theta})\right\}
\end{array}\right], \tilde{s}_{j} \triangleq\left[\begin{array}{c}
\Re\left\{s_{j}\right\} \\
\Im\left\{s_{j}\right\}
\end{array}\right], \\
\mathbf{V}_{j} \triangleq\left[\begin{array}{cc}
\mathbf{v}_{j}^{11} & \mathbf{v}_{j}^{12} \\
\mathbf{v}_{j}^{21} & \mathbf{v}_{j}^{22}
\end{array}\right], \tilde{n}_{k}=\left[\begin{array}{l}
\Re\left\{n_{k}\right\} \\
\Im\left\{n_{k}\right\}
\end{array}\right],
\end{array}
$$


under the following transformation:

$$
\left[\begin{array}{cc}
\Re\left\{\mathbf{w}_{1, j}\right\}+\Re\left\{\mathbf{w}_{2, j}\right\} & -\Im\left\{\mathbf{w}_{1, j}\right\}+\Im\left\{\mathbf{w}_{2, j}\right\} \\
\Im\left\{\mathbf{w}_{1, j}\right\}+\Im\left\{\mathbf{w}_{2, j}\right\} & \Re\left\{\mathbf{w}_{1, j}\right\}-\Re\left\{\mathbf{w}_{2, j}\right\}
\end{array}\right]=\mathbf{V}_{j} .
$$

This transform is indeed legitimate, since its inverse is given by

$$
\left[\begin{array}{ll}
\Re\left\{\mathbf{w}_{1, j}\right\} & \Im\left\{\mathbf{w}_{1, j}\right\} \\
\Re\left\{\mathbf{w}_{2, j}\right\} & \Im\left\{\mathbf{w}_{2, j}\right\}
\end{array}\right]=\frac{1}{2}\left[\begin{array}{cc}
\mathbf{v}_{j}^{11}+\mathbf{v}_{j}^{22} & \mathbf{v}_{j}^{21}-\mathbf{v}_{j}^{12} \\
\mathbf{v}_{j}^{11}-\mathbf{v}_{j}^{22} & \mathbf{v}_{j}^{21}+\mathbf{v}_{j}^{12}
\end{array}\right] .
$$

Furthermore, we have:

$$
\left\|\mathbf{w}_{j}\right\|^{2}=\frac{1}{2} \sum_{i=1}^{2} \sum_{\ell=1}^{2}\left\|\mathbf{v}_{j}^{i \ell}\right\|^{2},
$$

hence the power constraint (35b) for $\mathbf{w}$ is transferred to the following constraint

$$
\sum_{j=1}^{K}\left\|\mathbf{v}_{j}\right\|^{2} \leq 2 P
$$

for

$$
\mathbf{v}_{j} \triangleq \operatorname{vec}\left(\mathbf{V}_{j}\right)=\left[\begin{array}{c}
\mathbf{v}_{j}^{11} \\
\mathbf{v}_{j}^{21} \\
\mathbf{v}_{j}^{12} \\
\mathbf{v}_{j}^{22}
\end{array}\right] .
$$

For $\mathbf{v} \triangleq\left\{\mathbf{v}_{j}, j \in \mathcal{K}\right\}$, the problem (36) is equivalent to the problem

$$
\max _{\mathbf{v}, \boldsymbol{\theta}} \tilde{F}^{(\kappa)}(\mathbf{v}, \boldsymbol{\theta}) \triangleq \sum_{k=1}^{K} \gamma_{k}^{(\kappa)} \tilde{r}_{k}(\mathbf{v}, \boldsymbol{\theta}) \quad \text { s.t. }
$$

with

$$
\begin{aligned}
& \tilde{r}_{k}(\mathbf{v}, \boldsymbol{\theta})= \\
& \ln \left|I_{2}+\left[\overline{\mathcal{H}}_{k}(\boldsymbol{\theta}) \mathbf{V}_{k}\right]^{2}\left(\sum_{j \in \mathcal{K} \backslash\{k\}}\left[\overline{\mathcal{H}}_{k}(\boldsymbol{\theta}) \mathbf{V}_{j}\right]^{2}+\sigma I_{2}\right)^{-1}\right| .
\end{aligned}
$$

We propose the following alternating descent iterations at the $\kappa$-th round to generate a better feasible point $\left(w^{(\kappa+1)}, \theta^{(\kappa+1)}\right)$.

\section{A. Widely linear beamforming descent iteration}

We seek $w^{(\kappa+1)}$ such that

$$
F^{(\kappa)}\left(w^{(\kappa+1)}, \theta^{(\kappa)}\right)>F^{(\kappa)}\left(w^{(\kappa)}, \theta^{(\kappa)}\right) .
$$

Upon using (41) to define

$$
V_{j}^{(\kappa)} \triangleq\left[\begin{array}{cc}
\Re\left\{w_{1, j}^{(\kappa)}\right\}+\Re\left\{w_{2, j}^{(\kappa)}\right\} & -\Im\left\{w_{1, j}^{(\kappa)}\right\}+\Im\left\{w_{2, j}^{(\kappa)}\right\} \\
\Im\left\{w_{1, j}^{(\kappa)}\right\}+\Im\left\{w_{2, j}^{(\kappa)}\right\} & \Re\left\{w_{1, j}^{(\kappa)}\right\}-\Re\left\{w_{2, j}^{(\kappa)}\right\}
\end{array}\right]
$$

we have $v_{j}^{(\kappa)} \triangleq \operatorname{vec}\left(V_{j}^{(\kappa)}\right)$.

By using the inequality (14) for $\mathbf{V}=\overline{\mathcal{H}}_{k}\left(\theta^{(\kappa)}\right) \mathbf{V}_{k}, \mathbf{Y}=$ $\sum_{j \in \mathcal{K} \backslash\{k\}}\left[\overline{\mathcal{H}}_{k}\left(\theta^{(\kappa)}\right) \mathbf{V}_{j}\right]^{2}+\sigma I_{2}$, and $\bar{V}=\overline{\mathcal{H}}_{k}\left(\theta^{(\kappa)}\right) V_{k}^{(\kappa)}$, $\bar{Y}=Y_{k}^{(\kappa)} \triangleq \sum_{j \in \mathcal{K} \backslash\{k\}}\left[\overline{\mathcal{H}}_{k}\left(\theta^{(\kappa)}\right) V_{j}^{(\kappa)}\right]^{2}+\sigma I_{2} \succeq 0$, we obtain the following concave quadratic lower bounding function approximation of $\tilde{r}_{k}\left(\theta^{(\kappa)}, \mathbf{v}\right)$ :

$\tilde{r}_{k}\left(\mathbf{v}, \theta^{(\kappa)}\right) \geq \tilde{r}_{k}^{(\kappa)}(\mathbf{v})$

$$
\triangleq a_{k}^{(\kappa)}+2\left\langle B_{k}^{(\kappa)} \mathbf{V}_{k}\right\rangle-\left\langle C_{k}^{(\kappa)}, \sum_{j \in \mathcal{K}}\left[\overline{\mathcal{H}}_{k}\left(\theta^{(\kappa)}\right) \mathbf{V}_{j}\right]^{2}\right\rangle
$$

with $a_{k}^{(\kappa)} \triangleq \tilde{r}_{k}\left(v^{(\kappa)}, \theta^{(\kappa)}\right)-\left\langle\left[\overline{\mathcal{H}}_{k}\left(\theta^{(\kappa)}\right) V_{k}^{(\kappa)}\right]^{2}\left(Y_{k}^{(\kappa)}\right)^{-1}\right\rangle-$ $\sigma\left\langle C_{k}^{(\kappa)}\right\rangle, B_{k}^{(\kappa)} \triangleq\left(V_{k}^{(\kappa)}\right)^{H}\left(\overline{\mathcal{H}}_{k}\left(\theta^{(\kappa)}\right)\right)^{t}\left(Y_{k}^{(\kappa)}\right)^{-1}$

$\times \overline{\mathcal{H}}_{k}\left(\theta^{(\kappa)}\right)$, and $0 \prec C_{k}^{(\kappa)} \triangleq\left(Y_{k}^{(\kappa)}\right)^{-1}-$ $\left(Y_{k}^{(\kappa)}+\left[\overline{\mathcal{H}}_{k}\left(\theta^{(\kappa)}\right) V_{k}^{(\kappa)}\right]^{2}\right)^{-1}$.

Note that $\left\langle B_{k}^{(\kappa)} \mathbf{V}_{k}\right\rangle=\left\langle\operatorname{vec}\left(\left(B_{k}^{(\kappa)}\right)^{T}\right), \mathbf{v}_{k}\right\rangle$, and

$$
\begin{aligned}
& \left\langle C_{k}^{(\kappa)},\left[\overline{\mathcal{H}}_{k}\left(\theta^{(\kappa)}\right) \mathbf{V}_{j}\right]^{2}\right\rangle \\
& \quad=\left\|\operatorname{vec}\left(\left(C_{k}^{(\kappa)}\right)^{1 / 2} \overline{\mathcal{H}}_{k}\left(\theta^{(\kappa)}\right) \mathbf{V}_{j}\right)\right\|^{2} \\
& \quad=\left\|\left(I_{2} \otimes\left(\left(C_{k}^{(\kappa)}\right)^{1 / 2} \overline{\mathcal{H}}_{k}\left(\theta^{(\kappa)}\right)\right)\right) \operatorname{vec}\left(\mathbf{V}_{j}\right)\right\|^{2} \\
& =\operatorname{vec}^{T}\left(\mathbf{V}_{j}\right)\left[I_{2} \otimes\left(\overline{\mathcal{H}}_{k}^{T}\left(\theta^{(\kappa)}\right) C_{k}^{(\kappa)} \overline{\mathcal{H}}_{k}\left(\theta^{(\kappa)}\right)\right)\right] \operatorname{vec}\left(\mathbf{V}_{j}\right) \\
& \quad=\mathbf{v}_{j}^{T} \mathcal{Q}_{k}^{(\kappa)} \mathbf{v}_{j}
\end{aligned}
$$

for $\mathcal{Q}_{k}^{(\kappa)} \triangleq I_{2} \otimes\left(\overline{\mathcal{H}}_{k}^{T}\left(\theta^{(\kappa)}\right) C_{k}^{(\kappa)} \overline{\mathcal{H}}_{k}\left(\theta^{(\kappa)}\right)\right)$.

Thus, we have

$$
\begin{aligned}
& \sum_{k=1}^{K} \gamma_{k}^{(\kappa)} \tilde{r}_{k}^{(\kappa)}(\mathbf{w}) \\
= & \sum_{k=1}^{K} \gamma_{k}^{(\kappa)} a_{k}^{(\kappa)}+2 \sum_{k=1}^{K}\left\langle\gamma_{k}^{(\kappa)} \operatorname{vec}\left(\left(B_{k}^{(\kappa)}\right)^{T}\right), \mathbf{v}_{k}\right\rangle \\
& +\sum_{k=1}^{K} \sum_{j=1}^{K} \mathbf{v}_{j}^{T}\left(\gamma_{k}^{(\kappa)} \mathcal{Q}_{k}^{(\kappa)}\right) \mathbf{v}_{j} \\
= & \sum_{k=1}^{K} \gamma_{k}^{(\kappa)} a_{k}^{(\kappa)}+2 \sum_{k=1}^{K}\left\langle\gamma_{k}^{(\kappa)} \operatorname{vec}\left(\left(B_{k}^{(\kappa)}\right)^{T}\right), \mathbf{v}_{k}\right\rangle \\
& +\sum_{k=1}^{K} \mathbf{v}_{k}^{T}\left(\sum_{j=1}^{K} \gamma_{j}^{(\kappa)} \mathcal{Q}_{j}^{(\kappa)}\right) \mathbf{v}_{k} .
\end{aligned}
$$

We solve the following convex problem at the $\kappa$-th iteration to generate $v^{(\kappa+1)}$ :

$$
\max _{\mathbf{w}} \sum_{k=1}^{K} \gamma_{k}^{(\kappa)} \tilde{r}_{k}^{(\kappa)}(\mathbf{w}) \quad \text { s.t. } \quad(44)
$$

which similarly to (16) gives

$$
\tilde{F}^{(\kappa)}\left(v^{(\kappa+1)}, \theta^{(\kappa)}\right)>\tilde{F}^{(\kappa)}\left(v^{(\kappa)}, \theta^{(\kappa)}\right)
$$

as far as $v^{(\kappa+1)} \neq v^{(\kappa)}$.

Like (16), the problem (52) admits the following closedform solution

$$
v_{k}^{(\kappa+1)}=\left\{\begin{array}{r}
\left(\sum_{j=1}^{K} \gamma_{j}^{(\kappa)} \mathcal{Q}_{j}^{(\kappa)}\right)^{-1} \gamma_{k}^{(\kappa)} \operatorname{vec}\left(\left(B_{k}^{(\kappa)}\right)^{T}\right) \\
\text { if } \sum_{k=1}^{K}\left\|\left(\sum_{j=1}^{K} \gamma_{j}^{(\kappa)} \mathcal{Q}_{j}^{(\kappa)}\right)^{-1} \gamma_{k}^{(\kappa)} \operatorname{vec}\left(\left(B_{k}^{(\kappa)}\right)^{T}\right)\right\|^{2} \\
\quad \leq 2 P \\
\left(\sum_{j=1}^{K} \gamma_{j}^{(\kappa)} \mathcal{Q}_{j}^{(\kappa)}+\mu I_{M}\right)^{-1} \gamma_{k}^{(\kappa)} \operatorname{vec}\left(\left(B_{k}^{(\kappa)}\right)^{T}\right) \\
\text { otherwise, }
\end{array}\right.
$$


where $\mu>0$ is found by bisection such that $\sum_{k=1}^{K}\left\|\left(\sum_{j=1}^{K} \gamma_{j}^{(\kappa)} \mathcal{Q}_{j}^{(\kappa)}+\mu I_{M}\right)^{-1} \gamma_{k}^{(\kappa)} \operatorname{vec}\left(\left(B_{k}^{(\kappa)}\right)^{T}\right)\right\|^{2}=$ $2 P$.

By reconstructing $v_{j}^{i \ell,(\kappa+1)}, i=1,2$ and $\ell=1,2$, from $v_{j}^{(\kappa+1)}$ we use (42) to determine $w_{1, j}^{(\kappa+1)}$ and $w_{2, j}^{(\kappa+1)}$ :

$$
\begin{array}{r}
{\left[\begin{array}{ll}
\Re\left\{w_{1, j}^{(\kappa+1)}\right\} & \Im\left\{w_{1, j}^{(\kappa+1)}\right\} \\
\Re\left\{w_{1, j}^{(\kappa+1)}\right\} & \Im\left\{w_{1, j}^{(\kappa+1)}\right\}
\end{array}\right]=} \\
\frac{1}{2}\left[\begin{array}{cc}
v_{j}^{11,(\kappa+1)}+v_{j}^{22,(\kappa+1)} & v_{j}^{21,(\kappa+1)}-v_{j}^{12,(\kappa+1)} \\
v_{j}^{11,(\kappa+1)}-v_{j}^{22,(\kappa+1)} & v_{j}^{21,(\kappa+1)}+v_{j}^{12,(\kappa+1)}
\end{array}\right],
\end{array}
$$

which results in (48).

\section{B. Programmable reflecting elements' descent iteration}

We seek $\theta^{(\kappa+1)}$ such that

$$
F^{(\kappa)}\left(w^{(\kappa+1)}, \theta^{(\kappa+1)}\right)>F^{(\kappa)}\left(w^{(\kappa+1)}, \theta^{(\kappa)}\right) .
$$

By using the inequality (14) for $\mathbf{V}=\Lambda_{k}(\boldsymbol{\theta}) W_{k}^{(\kappa+1)}, \mathbf{Y}=$ $\sum_{j \in \mathcal{K} \backslash\{k\}}\left[\Lambda_{k}(\boldsymbol{\theta}) W_{j}^{(\kappa+1)}\right]^{2}+\sigma I_{2}$, and $\bar{V}=\Lambda_{k}\left(\theta^{(\kappa)}\right) W_{k}^{(\kappa+1)}$, $\bar{Y}=Y_{k}^{(\kappa+1)} \triangleq \sum_{j \in \mathcal{K} \backslash\{k\}}\left[\Lambda_{k}\left(\theta^{(\kappa)}\right) W_{j}^{(\kappa+1)}\right]^{2}+\sigma I_{2} \succeq 0$, we obtain the following concave quadratic lower bounding function approximation of $r_{k}\left(w^{(\kappa+1)}, \boldsymbol{\theta}\right)$ :

$$
\begin{aligned}
r_{k}\left(w^{(\kappa+1)}, \boldsymbol{\theta}\right) \geq & \tilde{r}_{k}^{(\kappa)}(\boldsymbol{\theta}) \\
\triangleq & \tilde{a}_{1 k}^{(\kappa)}+2 \Re\left\{\left\langle\tilde{B}_{k}^{(\kappa)} \Lambda_{k}(\boldsymbol{\theta}) W_{k}^{(\kappa+1)}\right\rangle\right\} \\
& -\left\langle\tilde{C}_{k}^{(\kappa)}, \sum_{j \in \mathcal{K}}\left[\Lambda_{k}(\boldsymbol{\theta}) W_{j}^{(\kappa+1)}\right]^{2}\right\rangle \\
= & \tilde{a}_{1 k}^{(\kappa)}+2 \Re\left\{\left\langle\tilde{B}_{k}^{(\kappa)} \Lambda_{k}(\boldsymbol{\theta}) W_{k}^{(\kappa+1)}\right\rangle\right\} \\
& -\left\langle\tilde{C}_{k}^{(\kappa)}, \Lambda_{k}(\boldsymbol{\theta}) \mathcal{W}_{k}^{(\kappa+1)}\left(\Lambda_{k}(\boldsymbol{\theta})\right)^{H}\right\rangle,
\end{aligned}
$$

$\begin{array}{lllll}\text { with } & \tilde{a}_{1 k}^{(\kappa)} & r_{k}\left(w^{(\kappa+1)}, \theta^{(\kappa)}\right) & - \\ \left\langle\left[\Lambda_{k}\left(\theta^{(\kappa)}\right) W_{k}^{(\kappa+1)}\right]^{2}\left(Y_{k}^{(\kappa+1)}\right)^{-1}\right\rangle & -\sigma\left\langle\tilde{C}_{k}^{(\kappa)}\right\rangle, & \tilde{B}_{k}^{(\kappa)} & \triangleq\end{array}$ $\left(W_{k}^{(\kappa+1)}\right)^{H}\left(\Lambda_{k}\left(\theta^{(\kappa)}\right)\right)^{H}$

$\left(Y_{k}^{(\kappa+1)}\right)^{-1} \in \mathbb{C}^{2 \times 2}, 0 \prec \tilde{C}_{k}^{(\kappa)} \triangleq\left(Y_{k}^{(\kappa+1)}\right)^{-1}-$ $\left(Y_{k}^{(\kappa+1)}+\left[\Lambda_{k}\left(\theta^{(\kappa)}\right) W_{k}^{(\kappa+1)}\right]^{2}\right)^{-1} \in \mathbb{C}^{2 \times 2}, \quad$ and $0 \prec \mathcal{W}_{k}^{(\kappa+1)} \triangleq \sum_{j \in \mathcal{K}}\left[W_{j}^{(\kappa+1)}\right]^{2}$.

For

$$
\mathcal{H}_{\mathrm{B}-\mathrm{k}} \triangleq\left[\begin{array}{cc}
\tilde{h}_{\mathrm{B}-\mathrm{k}} & 0_{1 \times M} \\
0_{1 \times M} & \tilde{h}_{\mathrm{B}-\mathrm{k}}^{*}
\end{array}\right],
$$

we can write

$$
\begin{gathered}
\mathcal{H}_{\mathrm{B}-\mathrm{k}}+\left[\begin{array}{cc}
\tilde{h}_{\mathrm{BR}-\mathrm{k}} \operatorname{diag}\left(e^{\jmath \boldsymbol{\theta}}\right) H_{\mathrm{B}-\mathrm{R}} & \Lambda_{k}(\boldsymbol{\theta}) \\
0_{1 \times M} & \tilde{h}_{\mathrm{R}-\mathrm{k}}^{*} \operatorname{diag}\left(e^{-\jmath \boldsymbol{\theta}}\right) H_{\mathrm{B}-\mathrm{R}}^{*}
\end{array}\right]= \\
\mathcal{H}_{\mathrm{B}-\mathrm{k}}+\sum_{n=1}^{N}\left[\begin{array}{cc}
\tilde{h}_{\mathrm{BR}-\mathrm{k}} \Psi_{n} H_{\mathrm{B}-\mathrm{R}} & 0_{1 \times M} \\
0_{1 \times M} & 0_{1 \times M}
\end{array}\right] e^{\jmath \theta_{n}} \\
\left.+\left[\begin{array}{cc}
0_{1 \times M} & 0_{1 \times M} \\
0_{1 \times M} & \tilde{h}_{\mathrm{R}-\mathrm{k}}^{*} \Psi_{n} H_{\mathrm{B}-\mathrm{R}}^{*}
\end{array}\right] e^{-\jmath \theta_{n}}\right]= \\
\mathcal{H}_{\mathrm{B}-\mathrm{k}}+\sum_{n=1}^{N}\left[\Gamma_{n} e^{\jmath \theta_{n}}+\Xi_{n} e^{-\jmath \theta_{n}}\right]
\end{gathered}
$$

with

$$
\begin{gathered}
\Gamma_{n} \triangleq\left[\begin{array}{cc}
\tilde{h}_{\mathrm{BR}-\mathrm{k}} \Psi_{n} H_{\mathrm{B}-\mathrm{R}} & 0_{1 \times M} \\
0_{1 \times M} & 0_{1 \times M}
\end{array}\right], n=1, \ldots, N, \\
\Xi_{n} \triangleq\left[\begin{array}{cc}
0_{1 \times M} & 0_{1 \times M} \\
0_{1 \times M} & \tilde{h}_{\mathrm{R}-\mathrm{k}}^{*} \Psi_{n} H_{\mathrm{B}-\mathrm{R}}^{*}
\end{array}\right], n=1, \ldots, N .
\end{gathered}
$$

By using the identity

$$
\Re\left\{a b^{*}\right\}=\Re\left\{a^{*} b\right\} \quad \forall a \in \mathbb{C}, b \in \mathbb{C},
$$

we arrive at:

$$
\begin{aligned}
& \Re\left\{\left\langle\tilde{B}_{k}^{(\kappa)} \Lambda_{k}(\boldsymbol{\theta}) W_{k}^{(\kappa+1)}\right\rangle\right\} \\
= & \tilde{a}_{2 k}^{(\kappa)}+\Re\left\{\sum_{n=1}^{N}\left(\hat{b}_{1 k}^{(\kappa)}(n) e^{\jmath \theta_{n}}+\hat{b}_{2 k}^{(\kappa)}(n) e^{-\jmath \theta_{n}}\right)\right\} \\
= & \tilde{a}_{2 k}^{(\kappa)}+\Re\left\{\sum_{n=1}^{N} \tilde{b}_{2 k}^{(\kappa)}(n) e^{\jmath \theta_{n}}\right\},
\end{aligned}
$$

for $\tilde{a}_{2 k}^{(\kappa)} \triangleq \quad \Re\left\{\left\langle\tilde{B}_{k}^{(\kappa)} \mathcal{H}_{\mathrm{B}-\mathrm{k}} W_{k}^{(\kappa+1)}\right\rangle\right\}, \quad \hat{b}_{1 k}^{(\kappa)}(n) \triangleq$ $\left\langle\tilde{B}_{k}^{(\kappa)} \Gamma_{n} W_{k}^{(\kappa+1)}\right\rangle, \quad \hat{b}_{2 k}^{(\kappa)}(n) \triangleq\left\langle\tilde{B}_{k}^{(\kappa)} \Xi_{n} W_{k}^{(\kappa+1)}\right\rangle, \quad$ and $\tilde{b}_{2 k}^{(\kappa)}(n)=\hat{b}_{1 k}^{(\kappa)}(n)+\left(\hat{b}_{2 k}^{(\kappa)}\right)^{*}(n), n=1, \ldots, N$.

Furthermore, we have (62), where $\tilde{a}_{3 k}^{(\kappa)} \triangleq\left\langle\tilde{C}_{k}^{(\kappa)}, \mathcal{H}_{\mathrm{B}-\mathrm{k}} \mathcal{W}_{k}^{(\kappa+1)} \mathcal{H}_{\mathrm{B}-\mathrm{k}}^{H}\right\rangle, \quad \tilde{b}_{3 k}^{(\kappa)}(n) \triangleq$ $\left.\left\langle\tilde{C}_{k}^{(\kappa)}, \mathcal{H}_{\mathrm{B}-\mathrm{k}} \mathcal{W}_{k}^{(\kappa+1)} \Gamma_{n}^{H}\right\rangle^{*}+\tilde{C}_{k}^{(\kappa)}, \mathcal{H}_{\mathrm{B}-\mathrm{k}} \mathcal{W}_{k}^{(\kappa+1)} \Xi_{n}^{H}\right\rangle$, $\mathcal{Q}_{11, k}^{(\kappa)}(n, m)=\left\langle\tilde{C}_{k}^{(\kappa)}, \Xi_{n} \mathcal{W}_{k}^{(\kappa+1)} \Xi_{m}^{H}\right\rangle, \quad \mathcal{Q}_{22, k}^{(\kappa)}(n, m)=$ $\left\langle\tilde{C}_{k}^{(\kappa)}, \Gamma_{m} \mathcal{W}_{k}^{(\kappa+1)} \Gamma_{n}^{H}\right\rangle, \mathcal{Q}_{12, k}^{(\kappa)}(n, m)=\left\langle\tilde{C}_{k}^{(\kappa)}\right.$, $\left.\Gamma_{n} \mathcal{W}_{k}^{(\kappa+1)} \Xi_{m}^{H}\right\rangle, n=1, \ldots, N ; m=1, \ldots, N$.

Let us define

$$
\begin{gathered}
\mathcal{Q}_{22, k}^{(\kappa)}+\mathcal{Q}_{11, k}^{(\kappa)}=\mathcal{Q}_{2, k}^{R,(\kappa)}+\jmath \mathcal{Q}_{2, k}^{I,(\kappa)}, \\
\mathcal{Q}_{2, k}^{R,(\kappa)} \in \mathbb{R}^{N \times N}, \mathcal{Q}_{2, k}^{I,(\kappa)} \in \mathbb{R}^{N \times N},
\end{gathered}
$$

where the matrix $\mathcal{Q}_{2, k}^{R,(\kappa)}$ is symmetric, while the matrix $\mathcal{Q}_{2, k}^{I,(\kappa)}$ is skew-symmetric because the matrix $\mathcal{Q}_{22, k}^{(\kappa)}+\mathcal{Q}_{11, k}^{(\kappa)}$ is Hermitian symmetric, and

$\mathcal{Q}_{12, k}^{(\kappa)}=\mathcal{Q}_{1, k}^{R,(\kappa)}+\jmath \mathcal{Q}_{1, k}^{I,(\kappa)}, \mathcal{Q}_{1, k}^{R,(\kappa)} \in \mathbb{R}^{N \times N}, \mathcal{Q}_{1, k}^{I,(\kappa)} \in \mathbb{R}^{N \times N}$.

Upon recalling that $e^{\jmath \theta}=\cos \theta+\jmath \sin \theta$, we have (63) and (64), whose proof is given in the Appendix.

Therefore, we have (65) for $\mathcal{Q}_{k, R}^{(\kappa)} \triangleq \mathcal{Q}_{2, k}^{R,(\kappa)}+\mathcal{Q}_{1, k}^{R,(\kappa)}+$ $\left(\mathcal{Q}_{1, k}^{R,(\kappa)}\right)^{T}, \mathcal{Q}_{k, C}^{(\kappa)} \triangleq-\mathcal{Q}_{2, k}^{I,(\kappa)}-\mathcal{Q}_{1, k}^{I,(\kappa)}-\left(\mathcal{Q}_{1, k}^{I,(\kappa)}\right)^{T}, \mathcal{Q}_{k, I}^{(\kappa)} \triangleq$ $\mathcal{Q}_{2, k}^{R,(\kappa)}-\mathcal{Q}_{1, k}^{R,(\kappa)}-\left(\mathcal{Q}_{1, k}^{R,(\kappa)}\right)^{T}$.

Combining (57), (61), (62), and (65) yields

$$
\begin{aligned}
\gamma_{k}^{(\kappa)} \tilde{r}^{(\kappa)}\left(w^{(\kappa+1)}, \boldsymbol{\theta}\right)= & \tilde{a}_{k}^{(\kappa)}+2 \Re\left\{\sum_{n=1}^{N} \tilde{b}_{k}^{(\kappa)}(n) e^{\jmath \theta_{n}}\right\} \\
& -\left[\begin{array}{c}
\cos \boldsymbol{\theta} \\
\sin \boldsymbol{\theta}
\end{array}\right]^{T} \mathcal{Q}_{k}^{(\kappa)}\left[\begin{array}{l}
\cos \boldsymbol{\theta} \\
\sin \boldsymbol{\theta}
\end{array}\right]
\end{aligned}
$$

with $\tilde{a}_{k}^{(\kappa)}=\gamma_{k}^{(\kappa)}\left(\tilde{a}_{1 k}^{(\kappa)}+2 \tilde{a}_{2 k}^{(\kappa)}-\tilde{a}_{3 k}^{(\kappa)}\right), \quad \tilde{b}_{k}^{(\kappa)}(n)=$ $\gamma_{k}^{(\kappa)}\left(\tilde{b}_{2 k}^{(\kappa)}(n)-\tilde{b}_{3 k}^{(\kappa)}(n)\right), \quad n=1, \ldots, N, \quad \mathcal{Q}_{k}^{(\kappa)}=$ $\gamma_{k}^{(\kappa)}\left[\begin{array}{cc}\mathcal{Q}_{k, R}^{(\kappa)} & \mathcal{Q}_{k, C}^{(\kappa)} \\ \left(\mathcal{Q}_{k, C}^{(\kappa)}\right)^{T} & \mathcal{Q}_{k, I}^{(\kappa)}\end{array}\right]$.

Therefore, we have:

$F^{(\kappa)}\left(w^{(\kappa+1)}, \boldsymbol{\theta}, \gamma^{(\kappa)}\right) \geq F_{c}^{(\kappa)}(\boldsymbol{\theta})$ 


$$
\begin{aligned}
& \left\langle\tilde{C}_{k}^{(\kappa)}, \Lambda_{k}(\boldsymbol{\theta}) \mathcal{W}_{k}^{(\kappa+1)} \Lambda_{k}^{H}(\boldsymbol{\theta})\right\rangle= \\
& \left\langle\tilde{C}_{k}^{(\kappa)},\left[\sum_{n=1}^{N}\left(\Gamma_{n} e^{\jmath \theta_{n}}+\Xi_{n} e^{-\jmath e^{\jmath \theta_{n}}}\right)+\mathcal{H}_{\mathrm{B}-\mathrm{k}}\right] \mathcal{W}_{k}^{(\kappa+1)}\left[\sum_{n=1}^{N}\left(\Gamma_{n}^{H} e^{-\jmath \theta_{n}}+\Xi_{n}^{H} e^{\jmath \theta_{n}}\right)+\mathcal{H}_{\mathrm{B}-\mathrm{k}}^{H}\right]\right\rangle= \\
& \left\langle\tilde{C}_{k}^{(\kappa)}, \mathcal{H}_{\mathrm{B}-\mathrm{k}} \mathcal{W}_{k}^{(\kappa+1)} \mathcal{H}_{\mathrm{B}-\mathrm{k}}^{H}\right\rangle+2 \Re\left\{\left\langle\tilde{C}_{k}^{(\kappa)}, \mathcal{H}_{\mathrm{B}-\mathrm{k}} \mathcal{W}_{k}^{(\kappa+1)} \sum_{n=1}^{N}\left(\Gamma_{n}^{H} e^{-\jmath \theta_{n}}+\Xi_{n}^{H} e^{\jmath \theta_{n}}\right)\right\}\right. \\
& +\left\langle\tilde{C}_{k}^{(\kappa)},\left[\sum_{n=1}^{N}\left(\Gamma_{n} e^{\jmath \theta_{n}}+\Xi_{n} e^{-\jmath \theta_{n}}\right)\right] \mathcal{W}_{k}^{(\kappa+1)}\left[\sum_{n=1}^{N}\left(\Gamma_{n}^{H} e^{-\jmath \theta_{n}}+\Xi_{n}^{H} e^{\jmath \theta_{n}}\right)\right]\right\rangle= \\
& \left\langle\tilde{C}_{k}^{(\kappa)}, \mathcal{H}_{\mathrm{B}-\mathrm{k}} \mathcal{W}_{k}^{(\kappa+1)} \mathcal{H}_{\mathrm{B}-\mathrm{k}}^{H}\right\rangle+2 \Re\left\{\sum_{n=1}^{N}\left(\left\langle\tilde{C}_{k}^{(\kappa)}, \mathcal{H}_{\mathrm{B}-\mathrm{k}} \mathcal{W}_{k}^{(\kappa+1)} \Gamma_{n}^{H}\right\rangle^{*}+\left\langle\tilde{C}_{k}^{(\kappa)}, \mathcal{H}_{\mathrm{B}-\mathrm{k}} \mathcal{W}_{k}^{(\kappa+1)} \Xi_{n}^{H}\right\rangle\right) e^{\jmath \theta_{n}}\right\} \\
& +\sum_{n=1}^{N} \sum_{m=1}^{N}\left\langle\tilde{C}_{k}^{(\kappa)}, \Gamma_{n} \mathcal{W}_{k}^{(\kappa+1)} \Gamma_{m}^{H}\right\rangle e^{\jmath \theta_{n}} e^{-\jmath \theta_{m}}+\sum_{n=1}^{N} \sum_{m=1}^{N}\left\langle\tilde{C}_{k}^{(\kappa)}, \Gamma_{n} \mathcal{W}_{k}^{(\kappa+1)} \Xi_{m}^{H}\right\rangle e^{\jmath \theta_{n}} e^{-\jmath \theta_{m}} \\
& +\sum_{n=1}^{N} \sum_{m=1}^{N}\left\langle\tilde{C}_{k}^{(\kappa)}, \Xi_{n} \mathcal{W}_{k}^{(\kappa+1)} \Gamma_{m}^{H}\right\rangle e^{-\jmath \theta_{n}} e^{-\jmath \theta_{m}}+\sum_{n=1}^{N} \sum_{m=1}^{N}\left\langle\tilde{C}_{k}^{(\kappa)}, \Xi_{n} \mathcal{W}_{k}^{(\kappa+1)} \Xi_{m}^{H}\right\rangle e^{-\jmath \theta_{n}} e^{-\jmath \theta_{m}}= \\
& \left.\tilde{a}_{3 k}^{(\kappa)}+2 \Re\left\{\sum_{n=1}^{N} \tilde{b}_{3 k}^{(\kappa)}(n) e^{\jmath \theta_{n}}\right\rangle\right\}+\left(e^{\jmath \boldsymbol{\theta}}\right)^{H} \mathcal{Q}_{22, k}^{(\kappa)} e^{\jmath \boldsymbol{\theta}}+\left(e^{\jmath \boldsymbol{\theta}}\right)^{T} \mathcal{Q}_{12, k}^{(\kappa)} e^{\jmath \boldsymbol{\theta}}+\left(e^{\jmath \boldsymbol{\theta}}\right)^{H}\left(\mathcal{Q}_{12, k}^{(\kappa)}\right)^{*} e^{-\jmath \boldsymbol{\theta}}+\left(e^{\jmath \boldsymbol{\theta}}\right)^{H} \mathcal{Q}_{11, k}^{(\kappa)} e^{\jmath \boldsymbol{\theta}},
\end{aligned}
$$

$$
\left(e^{\jmath \boldsymbol{\theta}}\right)^{H}\left(\mathcal{Q}_{22, k}^{(\kappa)}+\mathcal{Q}_{11, k}^{(\kappa)}\right) e^{\boldsymbol{\theta} \boldsymbol{\theta}}=\left[\begin{array}{c}
\cos \boldsymbol{\theta} \\
\sin \boldsymbol{\theta}
\end{array}\right]^{T}\left[\begin{array}{cc}
\mathcal{Q}_{2, k}^{R,(\kappa)} & -\mathcal{Q}_{2, k}^{I,(\kappa)} \\
\mathcal{Q}_{2, k}^{I,(\kappa)} & \mathcal{Q}_{2, k}^{R,(\kappa)}
\end{array}\right]\left[\begin{array}{c}
\cos \boldsymbol{\theta} \\
\sin \boldsymbol{\theta}
\end{array}\right]
$$

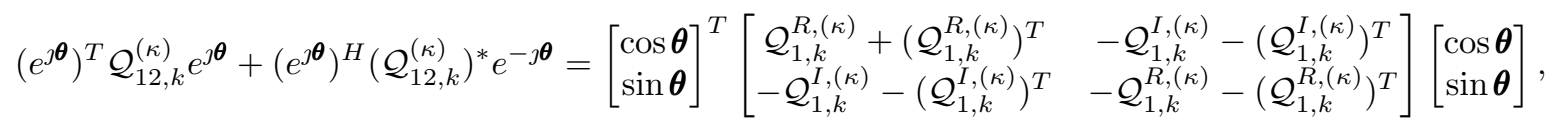

$$
\left(e^{\jmath \boldsymbol{\theta}}\right)^{H}\left(\mathcal{Q}_{22, k}^{(\kappa)}+\mathcal{Q}_{11, k}^{(\kappa)}\right) e^{\boldsymbol{\theta} \boldsymbol{\theta}}+\left(e^{\jmath \boldsymbol{\theta}}\right)^{T} \mathcal{Q}_{12, k}^{(\kappa)} e^{\jmath \boldsymbol{\theta}}+\left(e^{\jmath \boldsymbol{\theta}}\right)^{H}\left(\mathcal{Q}_{12, k}^{(\kappa)}\right)^{*} e^{-\jmath \boldsymbol{\theta}}=\left[\begin{array}{c}
\cos \boldsymbol{\theta} \\
\sin \boldsymbol{\theta}
\end{array}\right]^{T}\left[\begin{array}{cc}
\mathcal{Q}_{k, R}^{(\kappa)} & \mathcal{Q}_{k, C}^{(\kappa)} \\
\left(\mathcal{Q}_{k, C}^{(\kappa)}\right)^{T} & \mathcal{Q}_{k, I}^{(\kappa)}
\end{array}\right]\left[\begin{array}{c}
\cos \boldsymbol{\theta} \\
\sin \boldsymbol{\theta}
\end{array}\right]
$$

$$
\triangleq \tilde{a}^{(\kappa)}+2 \Re\left\{\sum_{n=1}^{N} \tilde{b}^{(\kappa)}(n) e^{\jmath \theta_{n}}\right\}-\left[\begin{array}{c}
\cos \boldsymbol{\theta} \\
\sin \boldsymbol{\theta}
\end{array}\right]^{T} \mathcal{Q}^{(\kappa)}\left[\begin{array}{c}
\cos \boldsymbol{\theta} \\
\sin \boldsymbol{\theta}
\end{array}\right]
$$

for $\tilde{a}^{(\kappa)}=\sum_{k=1}^{K} \tilde{a}_{k}^{(\kappa)}, \tilde{b}^{(\kappa)}(n)=\sum_{k=1}^{K} \tilde{b}_{k}^{(\kappa)}(n), n=$ $1, \ldots, N$, and $\mathcal{Q}^{(\kappa)}=\sum_{k=1}^{K} \mathcal{Q}_{k}^{(\kappa)}=\left[\begin{array}{cc}\mathcal{Q}_{R}^{(\kappa)} & \mathcal{Q}_{C}^{(\kappa)} \\ \left(\mathcal{Q}_{C}^{(\kappa)}\right)^{T} & \mathcal{Q}_{I}^{(\kappa)}\end{array}\right]$, with $\mathcal{Q}_{R}^{(\kappa)}=\sum_{k=1}^{K} \mathcal{Q}_{k, R}^{(\kappa)}, \mathcal{Q}_{C}^{(\kappa)}=\sum_{k=1}^{K} \mathcal{Q}_{k, C}^{(\kappa)}, \mathcal{Q}_{I}^{(\kappa)}=$ $\sum_{k=1}^{K} \mathcal{Q}_{k, I}^{(\kappa)}$.

Furthermore, we have (68). Now, using the formula

$$
\alpha^{R,(\kappa)}(n) \cos \boldsymbol{\theta}_{n}+\alpha^{I,(\kappa)}(n) \sin \boldsymbol{\theta}_{n}=\Re\left\{\beta(n) e^{\jmath \boldsymbol{\theta}_{n}}\right\}
$$

for $\beta(n)=\sqrt{\left(\alpha^{R,(\kappa)}(n)\right)^{2}+\left(\alpha^{I,(\kappa)}(n)\right)^{2}} e^{-\jmath \gamma(n)}$ , where $\gamma(n)$ is such that $[\cos \gamma(n), \sin \gamma(n)]=$ $\left[\alpha^{R,(\kappa)}(n), \alpha^{I,(\kappa)}(n)\right] / \sqrt{\left[\alpha^{R,(\kappa)}(n)\right]^{2}+\left[\alpha^{I,(\kappa)}(n)\right]^{2}}, \quad$ we can rewrite (68) by

$$
\left.\tilde{F}_{c}^{(\kappa)}(\boldsymbol{\theta})=\tilde{\tilde{a}}^{(\kappa)}+2 \Re\left\{\sum_{n=1}^{N} \tilde{b}^{(\kappa)}(n) e^{\jmath \theta_{n}}\right\rangle\right\}
$$

$$
\begin{aligned}
& -2 \sum_{n=1}^{N} \Re\left\{\beta(n) e^{\jmath \boldsymbol{\theta}_{n}}\right\} \\
= & \tilde{\tilde{a}}^{(\kappa)}+2 \sum_{n=1}^{N} \Re\left\{\left(\tilde{b}^{(\kappa)}(n)-\beta(n)\right) e^{\jmath \boldsymbol{\theta}_{n}}\right\}
\end{aligned}
$$

Accordingly, we solve the following convex problem at the $\kappa$-th iteration to generate $\theta^{(\kappa+1)}$ :

$$
\max _{\boldsymbol{\theta}} \tilde{F}_{c}^{(\kappa)}(\boldsymbol{\theta}) .
$$

Like (29), its optimal solution is given in closed-form by

$$
\theta_{n}^{(\kappa+1)}=-\angle\left(\tilde{b}^{(\kappa)}(n)-\beta(n)\right), n=1, \ldots, N .
$$

It follows from (68) that $F^{(\kappa)}\left(w^{(\kappa+1)}, \theta^{(\kappa+1)}\right) \geq$ $F_{c}^{(\kappa)}\left(\theta^{(\kappa+1)}\right) \geq \tilde{F}_{c}^{(\kappa)}\left(\theta^{(\kappa+1)}\right)>\tilde{F}_{c}^{(\kappa)}\left(\theta^{(\kappa)}\right)=F_{c}^{(\kappa)}\left(\theta^{(\kappa)}\right)=$ $F^{(\kappa)}\left(w^{(\kappa+1)}, \theta^{(\kappa)}\right)$, confirming (56), so $\theta^{(\kappa+1)}$ is a better feasible point than $\theta^{(\kappa)}$. 


$$
\begin{aligned}
F_{c}^{(\kappa)}(\boldsymbol{\theta})= & \left.\tilde{a}^{(\kappa)}+2 \Re\left\{\sum_{n=1}^{N} \tilde{b}^{(\kappa)}(n) e^{\jmath \theta_{n}}\right\rangle\right\}-\left[\begin{array}{c}
\cos \boldsymbol{\theta} \\
\sin \boldsymbol{\theta}
\end{array}\right]^{T}\left(\mathcal{Q}^{(\kappa)}-\lambda_{\max }\left(\mathcal{Q}^{(\kappa)}\right) I_{2 N}\right)\left[\begin{array}{c}
\cos \boldsymbol{\theta} \\
\sin \boldsymbol{\theta}
\end{array}\right] \\
& -\lambda_{\max }\left(\mathcal{Q}^{(\kappa)}\right) N \\
\geq & \left.\tilde{a}^{(\kappa)}+2 \Re\left\{\sum_{n=1}^{N} \tilde{b}^{(\kappa)}(n) e^{\jmath \theta_{n}}\right\rangle\right\}-2\left[\begin{array}{c}
\cos \theta^{(\kappa)} \\
\sin \theta^{(\kappa)}
\end{array}\right]^{T}\left(\mathcal{Q}^{(\kappa)}-\lambda_{\max }\left(\mathcal{Q}^{(\kappa)}\right) I_{2 N}\right)\left[\begin{array}{c}
\cos \boldsymbol{\theta} \\
\sin \boldsymbol{\theta}
\end{array}\right] \\
& +\left[\begin{array}{l}
\cos \theta^{(\kappa)} \\
\sin \theta^{(\kappa)}
\end{array}\right]^{T}\left(\mathcal{Q}^{(\kappa)}-\lambda_{\max }\left(\mathcal{Q}^{(\kappa)}\right) I_{2 N}\right)\left[\begin{array}{l}
\cos \theta^{(\kappa)} \\
\sin \theta^{(\kappa)}
\end{array}\right]-\lambda_{\max }\left(\mathcal{Q}^{(\kappa)}\right) N \\
= & \left.\tilde{\tilde{a}}^{(\kappa)}+2 \Re\left\{\sum_{n=1}^{N} \tilde{b}^{(\kappa)}(n) e^{\theta_{n}}\right\rangle\right\}-2 \sum_{n=1}^{N}\left(\alpha^{R,(\kappa)}(n) \cos \boldsymbol{\theta}_{n}+\alpha^{I,(\kappa)}(n) \sin \boldsymbol{\theta}_{n}\right) \\
\triangleq & \tilde{F}_{c}^{(\kappa)}(\boldsymbol{\theta}),
\end{aligned}
$$

with

$$
\begin{gathered}
\tilde{\tilde{a}}^{(\kappa)}=\tilde{a}^{(\kappa)}-\left[\begin{array}{c}
\cos \theta^{(\kappa)} \\
\sin \theta^{(\kappa)}
\end{array}\right]^{T} \mathcal{Q}^{(\kappa)}\left[\begin{array}{l}
\cos \theta^{(\kappa)} \\
\sin \theta^{(\kappa)}
\end{array}\right]-2 \lambda_{\max }\left(\mathcal{Q}^{(\kappa)}\right) N \\
\alpha^{R,(\kappa)}=\left(\theta^{R,(\kappa)}\right)^{T}\left(\mathcal{Q}_{R}^{(\kappa)}-\lambda_{\max }\left(\mathcal{Q}^{(\kappa)}\right) I_{N}\right)+\left(\theta^{I,(\kappa)}\right)^{T}\left(\mathcal{Q}_{C}^{(\kappa)}\right)^{T} \in \mathbb{R}^{1 \times N} \\
\alpha^{I,(\kappa)}=\left(\theta^{R,(\kappa)}\right)^{T}\left(\mathcal{Q}_{C}^{(\kappa)}\right)+\left(\theta^{I,(\kappa)}\right)^{T}\left(\mathcal{Q}_{I}^{(\kappa)}-\lambda_{\max }\left(\mathcal{Q}^{(\kappa)}\right) I_{N}\right) \in \mathbb{R}^{1 \times N}
\end{gathered}
$$

\section{Improper Gaussian Signaling Geometric Mean Rate Opti- mization}

All other exiting algorithms, which solve convex problems and iteratively at a high complexity are very sensitive to the problem sizes. However, our algorithms iterate using closedform expressions, hence their complexity is low. Algorithm 2 provides the pseudo-code for the proposed computational procedure for the solution of (36).

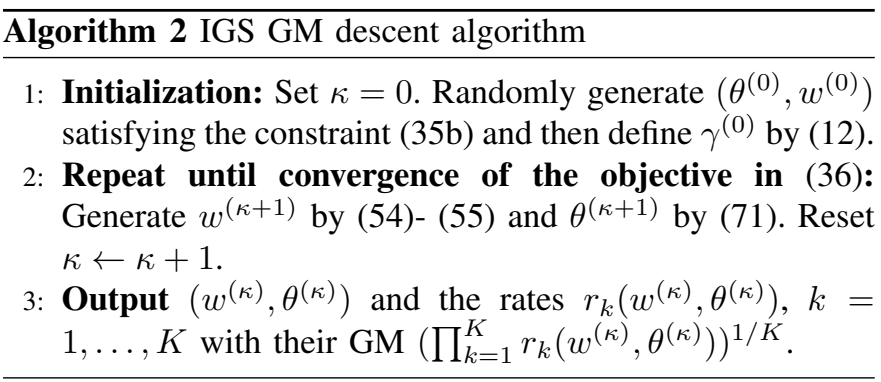

\section{NUMERICAL EXAMPLES}

This section evaluates the efficiency of the proposed algorithms by numerical examples. Table II provides the numerical values of the main parameters taken from [21], [34] for numerical characterization. Furthermore, the elements of the BS-to-RIS LoS channel matrix are generated by $\left[H_{\mathrm{B}-\mathrm{R}}\right]_{n, m}=$ $e^{j \pi\left((n-1) \sin \bar{\theta}_{n} \sin \bar{\phi}_{n}+(m-1) \sin e^{\jmath \theta_{n}} \sin \phi_{n}\right)}$, where $e^{j \theta_{n}}$ and $\phi_{n}$ are uniformly distributed as $e^{\jmath \theta_{n}} \sim \mathcal{U}(0, \pi)$ and $\phi_{n} \sim$ $\mathcal{U}(0,2 \pi)$, respectively, and $\bar{\theta}_{n}=\pi-\theta_{n}$ and $\bar{\phi}_{n}=\pi+\phi_{n}$ [21]. The normalized small-scale fading channel $h_{\mathrm{B}-\mathrm{k}}$ spanning from the BS to UE $k$ follows the classic Rayleigh distribution, while the small-scale fading channel gain $h_{\mathrm{R}-\mathrm{k}}$ of the RIS to UE $k$ obeys Rician distribution with a K-factor of 3 . The spatial correlation matrix is given by $\left[\mathbf{R}_{\mathrm{R}-\mathrm{k}}\right]_{n, n^{\prime}}=e^{j \pi\left(n-n^{\prime}\right) \sin \tilde{\phi}_{k} \sin \tilde{\theta}_{k}}$, where $\tilde{\phi}_{k}$ and $\tilde{\theta}$ are the azimuth and elevation angle for UE $k$, respectively. Unless otherwise stated, $P=20 \mathrm{dBm}$ and $N=100$ are used. The results are multiplied by $\log _{2} e$ to convert the unit nats/sec into the unit bps/Hz. The convergence tolerance of the proposed algorithms is set to $10^{-3}$. For computational stability, $\gamma_{k}^{(\kappa)}$ in (12) is scaled as

$$
\gamma_{k}^{(\kappa)} \rightarrow \frac{\gamma_{k}^{(\kappa)}}{\min _{j=1, \ldots, K} \gamma_{j}^{(\kappa)}}, k=1, \ldots, K .
$$

For the setup of Fig. 1 the BS and the RIS are deployed at the coordinates of $(40,0,25)$ and $(0,60,40)$ in the threedimensional (3D) space, while $K=10$ UEs are randomly placed in a $(120 m \times 120 m)$ area right of the BS and RIS. In what follows, we refer to SR-PGS and SR-IGS as the SR under PGS and IGS, which are achieved by iterating (18) and (29), and (54) and (55) with $\gamma_{k}^{(\kappa)} \equiv 1$. Their stand-alone counter-parts dispensing with the RIS are referred by SRPGS w/t RIS and SR-IGS w/t RIS, which are achieved by iterating (18) and and (54) with $\gamma_{k}^{(\kappa)} \equiv 1$ in the corresponding stand-alone models. Another pair of counter-parts labelled by SR-PGS-RIS w. random $\boldsymbol{\theta}$ and SR-IGS-RIS w. random $\boldsymbol{\theta}$ represent the SR with the PREs randomly selected, which correspond to iterating (18) and (54) under a fixed $\theta^{(\kappa)}=\bar{\theta}$ with $\gamma_{k}^{(\kappa)} \equiv 1$. Finally, GM-PGS-RIS and GM-IGS-RIS represent to the achievable GMs under PGS and IGS, which are computed by Algorithm 1 and 2 .

Fig. 2 plots the SR performance versus the number $M$ of antennas at the BS. The SR-PGS and SR-IGS are only slightly better than their counter-parts SR-IGS w/t RIS and SR-PGS, because the direct channel $\tilde{h}_{\mathrm{B}-\mathrm{k}}$ spanning from the BS to UE $k$ is much stronger than the reflected channel $\tilde{h}_{\mathrm{R}-\mathrm{k}} \mathbf{R}_{\mathrm{R}-\mathrm{k}}^{1 / 2}\left(e^{\jmath \boldsymbol{\theta}}\right) \tilde{H}_{\mathrm{B}-\mathrm{R}}$. The performance margin becomes wider with $M$ increased. Furthermore, SR-PGS approaches SRIGS for $M \geq K$ in Fig. 2. 
TABLE II: Major parameters setup

\begin{tabular}{|l|r|}
\hline Parameter & Numerical value \\
\hline BS-to-RIS path-loss $\beta_{\mathrm{B}-\mathrm{R}}$ at the distance $d_{\mathrm{B}-\mathrm{R}}$ in (4) & $G_{\mathrm{BS}}+G_{\mathrm{RIS}}-35.9-22 \log _{10}\left(d_{\mathrm{B}-\mathrm{R}}\right)$ (in $\left.\mathrm{dB}\right)$ \\
RIS-to-UE path-loss $\beta_{\mathrm{R}-\mathrm{k}}$ at the distance $d_{\mathrm{R}-\mathrm{k}}$ in (4) & $G_{\mathrm{RIS}}-33.05-30 \log _{10}\left(d_{\mathrm{R}-\mathrm{k}}\right)$ (in dB) \\
BS-to-UE Path-loss $\beta_{\mathrm{B}-\mathrm{k}}$ at the distance $d_{\mathrm{B}-\mathrm{k}}$ in (4) & $G_{\mathrm{BS}}-33.05-36.7 \log _{10}\left(d_{\mathrm{B}-\mathrm{k}}\right)$ (in dB) \\
Antenna gain $G_{\mathrm{BS}}$ of the BS and $G_{\mathrm{RIS}}$ of the RIS elements & $1 \mathrm{MHz}$ \\
Bandwidth & $-174 \mathrm{dBm} / \mathrm{Hz}$ \\
Noise power density & -174 \\
\hline
\end{tabular}

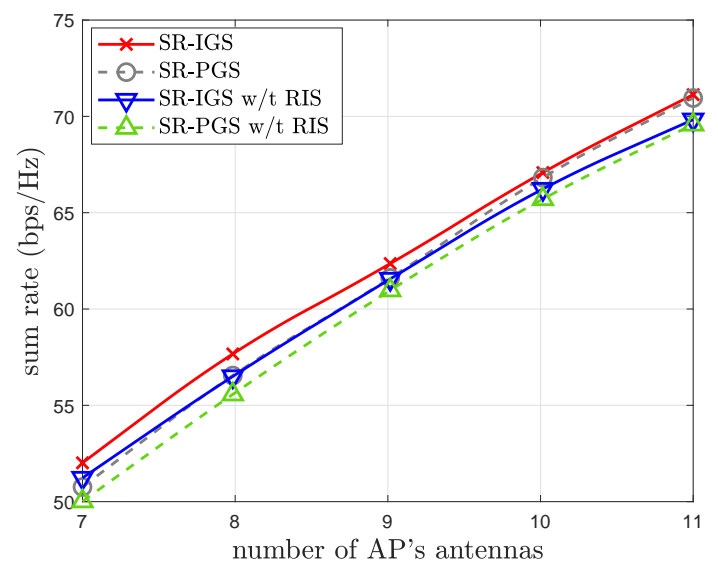

Fig. 2: SR versus the number of antennas $M$.

Next, Fig. 3 portrays a rate distribution pattern for $(M, N, P)=(9,100, P=20 \mathrm{dBm})$. Observe in the figure that only GM-IGS and GM-PGS are capable of avoiding the assignment of zero rate, hence demonstrating its superiority.

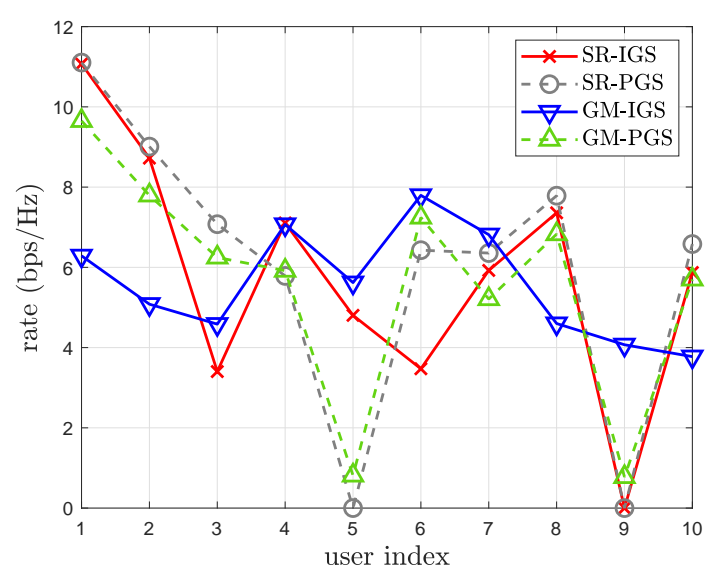

Fig. 3: Rate distribution for $M=9$.

To substantiate this fact, Table III provides the average number of zero-rate users (ZR-UEs) for the optimization schemes considered under different number of antennas $M$. For SR-IGS and SR-PGS, the number of ZR-UEs increases when $M$ is reduced. SR-PGS results in more ZR-UEs than SR-IGS, while there are no ZR-UEs in GM-IGS and GMPGS, confirming that both of them are beneficial in providing the adequate rates to all users.

Furthermore, we also examine the resultant ratio of the minimum rate and maximum rate (min-rate/max-rate) and the resultant rate-variance of these schemes versus the number of antennas, $M$. Fig. 4 shows that both GM-PGS and GMIGS produce min-rate/max-rates that are substantially higher than that of SR-PGS and SR-IGS. SR-IGS produces higher min-rate/max-rates than SR-PGS does. Fig. 4 also shows the min-rate/max-rate of SR-PGS remains zero for $M<K$ since there are always some ZR-UEs. Furthermore, upon increasing the number of AP antennas, both the min-rate and the maxrate both are improved due to the increased benefit of spatial diversity, but the value of min-rate /max-rate is not necessary a monotonic function of the number of AP antennas. In Fig. 5 , the rate variance of SR-PGS is seen to be twice of that by its IGS counter SR-IGS at $M=7$. The discrepancy becomes narrower upon increasing $M$ and it is closer to zero for $M=11$. The rate-variances are beneficially reduced by the GM-maximization based schemes GM-IGS and GM-PGS. Both Fig. 4 and Fig. 5 indicate the advantages of IGS over PGS both in terms of SR and GM maximization. Fig. 6 shows the

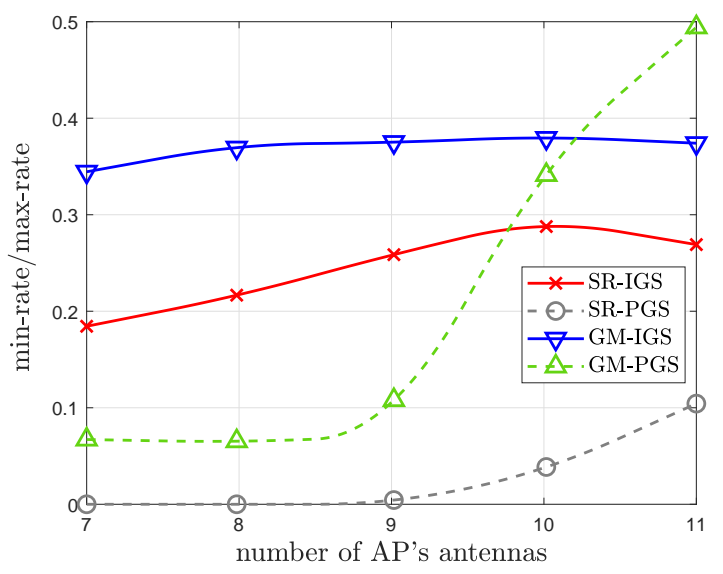

Fig. 4: Min-rate/max-rate versus the number of antennas $M$.

GM rates. As expected, GM-IGS and GM-PGS produce much better GM rate than that of SR-IGS and SR-PGS. Note that GM-PGS has better GM rates than GM-IGS for $M>K$ due to the well-known capability of PGS to mitigate the multi-user interference, when the number of transmit antennas is higher than the number of users.

We also consider another scenario as illustrated by Fig. 7 , where the direct signal path between the BS and users is blocked, i.e. we have $h_{\mathrm{B}-\mathrm{k}} \equiv 0$ in (2) and (4). The distances between the BS and users becomes slightly smaller upon deploying the BS at the coordinates $(20,0,25)$ and the RIS at the coordinates $(0,30,40)$. In this scenario, $K=10$ UEs are randomly placed in a $(60 \mathrm{~m} \times 60 \mathrm{~m})$ area right of the BS and RIS. 
TABLE III: The average number of ZR-UEs versus $M$

\begin{tabular}{|c|c|c|c|c|}
\hline Number of antennas & SR-IGS & SR-PGS & GM-IGS & GM-PGS \\
\hline $\mathrm{M}=7$ & 0.33 & 3.13 & 0 & 0 \\
\hline $\mathrm{M}=8$ & 0.23 & 2.37 & 0 & 0 \\
\hline $\mathrm{M}=9$ & 0.17 & 1.64 & 0 & 0 \\
\hline $\mathrm{M}=10$ & 0 & 1.10 & 0 & 0 \\
\hline $\mathrm{M}=11$ & 0 & 0.72 & 0 & 0 \\
\hline
\end{tabular}

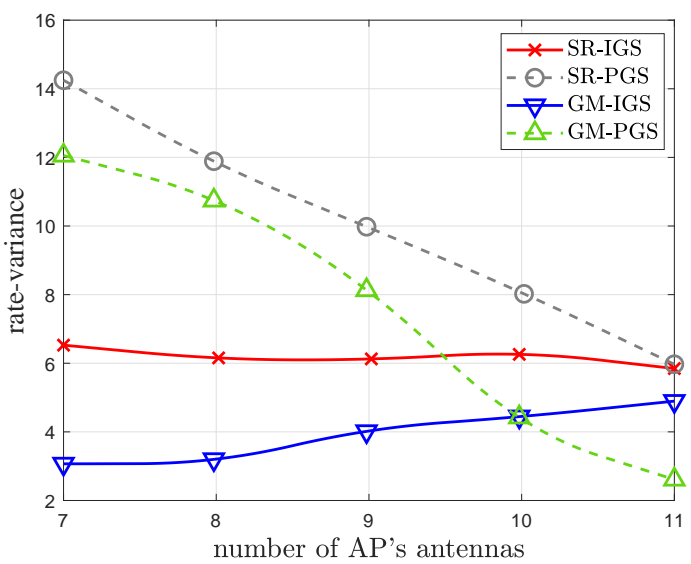

Fig. 5: Rate-variance versus the number of antennas $M$.

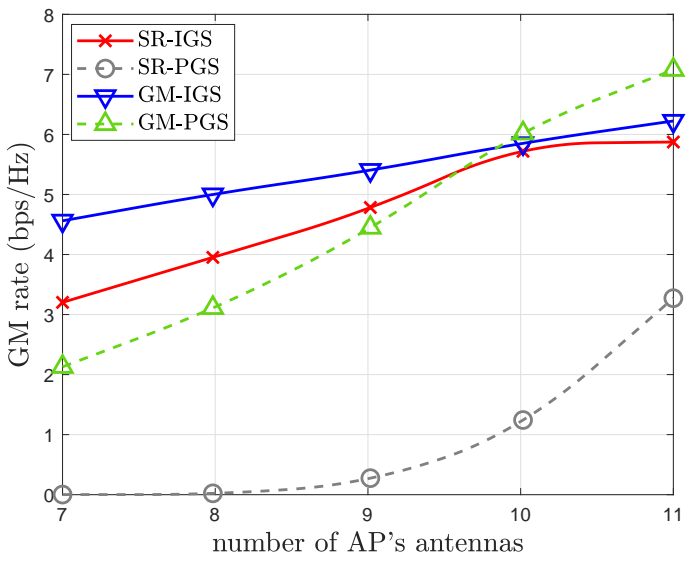

Fig. 6: GM versus the number of antennas $M$.

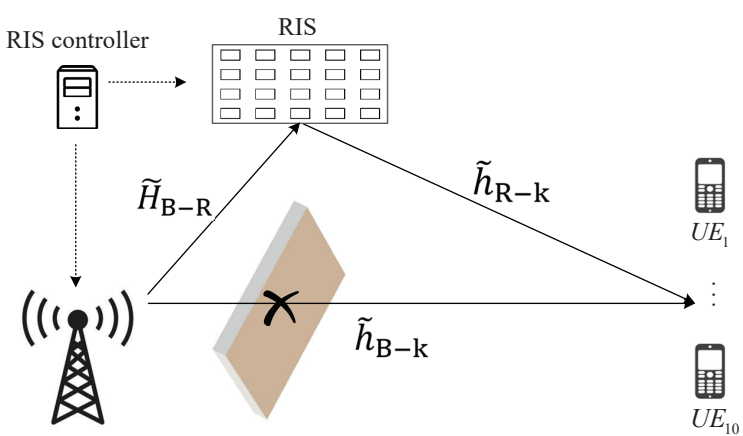

Fig. 7: System model

Fig. 8 portrays the SR versus $M$, where SR-IGS out- performs SR-PGS. Furthermore, both the former and the latter substantially outperform their counter-parts SR-IGS w. random $\theta$ and SR-PGS $\mathbf{w}$. random $\theta$ operating without an RIS.

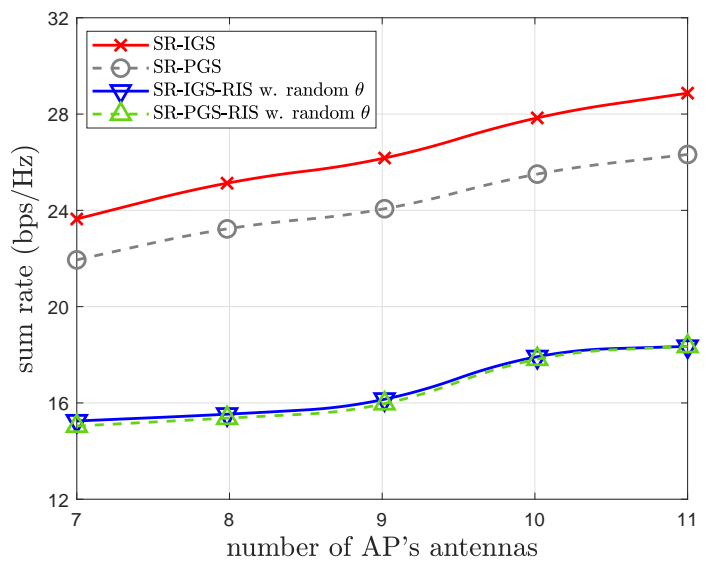

Fig. 8: SR versus the number of antennas $M$.

Similarly to Fig. 3, Fig. 9 shows a typical user rate distribution, where both the GM maximization based GM-IGS and GM-PGS schemes assign more transmit power to the users having worse channel conditions for achieving fair rate distributions.

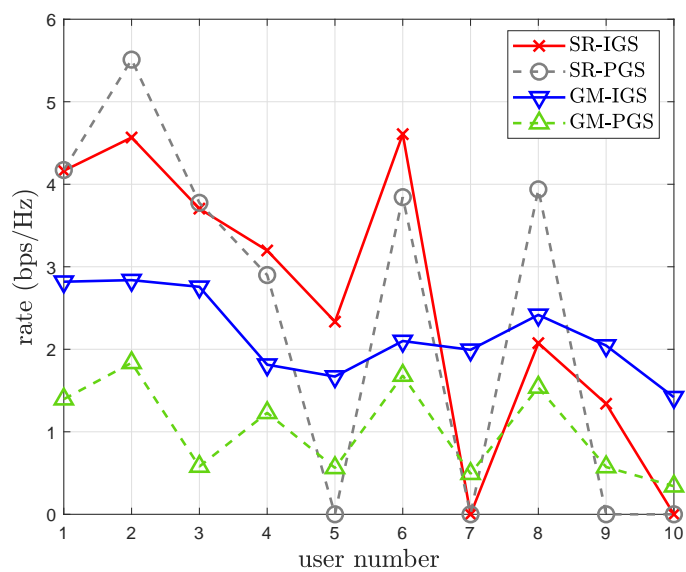

Fig. 9: User rate distribution for $M=9$.

Table IV shows the average number of ZR-UEs versus $M$, demonstrating that the number of ZR-UEs for both SR-IGS and SR-PGS is higher than 3, with that of SR-PGS having higher than that of SR-IGS. As expected, there are no ZR-UEs for GM-IGS and GM-PGS. 
TABLE IV: The average number of ZR-UEs versus the number of antennas $M$

\begin{tabular}{|c|c|c|c|c|}
\hline Number of antennas & SR-IGS & SR-PGS & GM-IGS & GM-PGS \\
\hline $\mathrm{M}=7$ & 3.97 & 5.40 & 0 & 0 \\
\hline $\mathrm{M}=8$ & 3.63 & 4.79 & 0 & 0 \\
\hline $\mathrm{M}=9$ & 3.20 & 5.61 & 0 & 0 \\
\hline $\mathrm{M}=10$ & 3.30 & 4.13 & 0 & 0 \\
\hline $\mathrm{M}=11$ & 3.11 & 3.82 & 0 & 0 \\
\hline
\end{tabular}

Fig. 10 and Fig. 11 plot the min-rate/max-rate and ratevariance versus $M$, respectively. The min-rate/max-rate of SRIGS and SR-PGS remains zero for the practical range of $M \in\{7, \ldots, 11\}$. Furthermore, GM-IGS has a better performance than GM-PGS . Fig. 11 shows that the rate variance is substantially improved by the GM-based maximization, where GM-IGS results in much better rate variance than GM-PGS. The advantage of GM rate maximization based IGS becomes quite convincing.

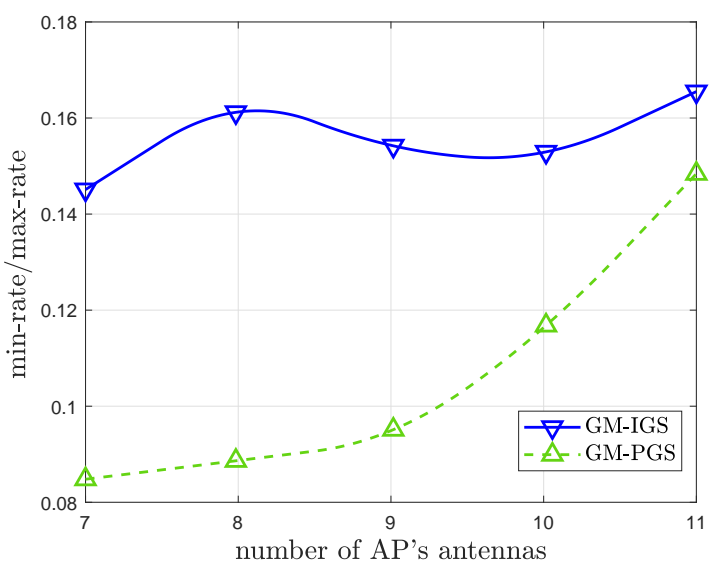

Fig. 10: Min-rate/max-rate versus the number of antennas $M$.

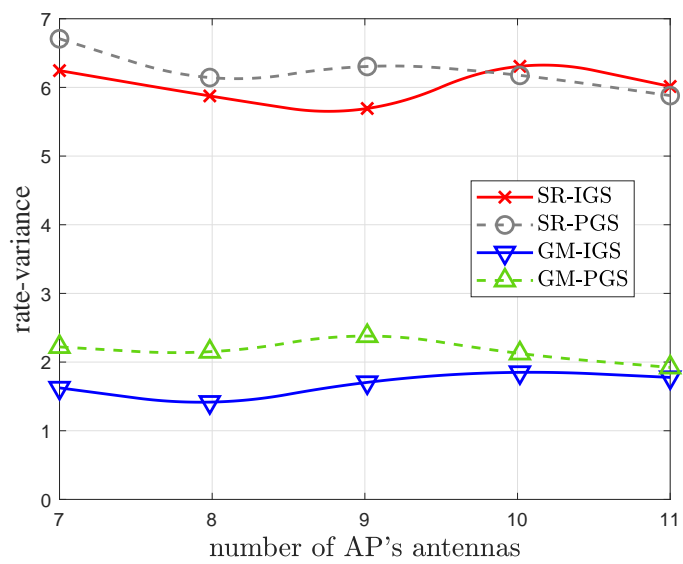

Fig. 11: Rate-variance versus the number of antennas $M$.

Finally, Fig. 12 plots the GM rate versus $M$, which remains zero for both SR-IGS and SR-PGS for $M \in\{7, \ldots, 11\}$, because there are ZR-UEs. The performance of GM-PGS gets closer to that of GM-IGS for $M \geq K$. The advantage of rates GM maximization based IGS is well justified in above results.

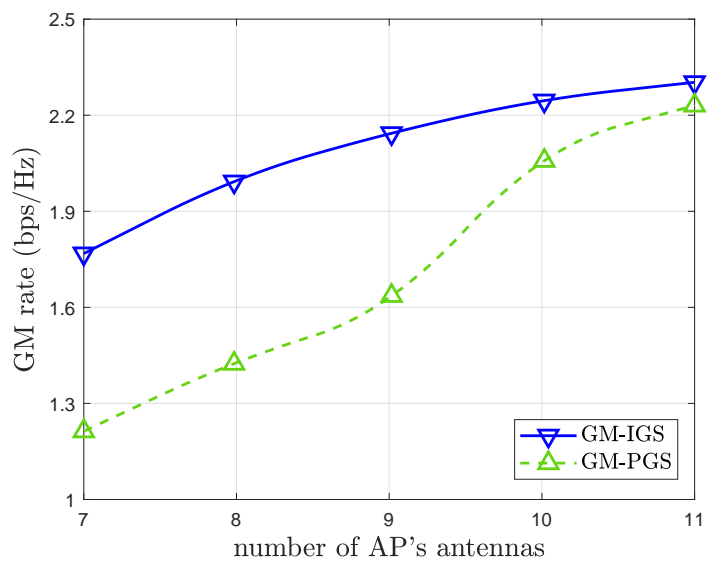

Fig. 12: GM rate versus the number of antennas $M$.

\section{CONCLUSIONS}

We proposed to maximize the geometric mean (GM) of the users' rates for the sake of maintaining a uniform quality-ofservice for the downlink users of an RIS-aid communication network. The computationally intractable unit modulus constraint imposed on the programmable reflecting coefficients has been eliminated by directly optimizing their argument. The problem of maximizing the users' GM rate has been solved by the proposed alternating descent iterations leading to a closedform solution for the associated convex problems and thus it is computationally efficient. The numerical examples provided have shown a substantially improved rate-fairness amongst the users. Extension of the GM maximization-based approach to multi-carrier communication is under our current study. Its extension to the quantized RIS-aided communication is also interesting and deserves a separate study in our future research.

\section{APPENDIX: PROOF OF (63) AND (64)}

Note that

$$
\mathcal{Q}_{22, k}^{(\kappa)}+\mathcal{Q}_{11, k}^{(\kappa)}=\mathcal{Q}_{2, k}^{R,(\kappa)}+\jmath \mathcal{Q}_{2, k}^{I,(\kappa)}
$$

and $\mathcal{Q}_{2, k}^{R,(\kappa)}$ is symmetric, while $\mathcal{Q}_{2, k}^{I,(\kappa)}$ is anti-symmetric $\left(\mathcal{Q}_{2, k}^{I,(\kappa)}=-\left(\mathcal{Q}_{2, k}^{I,(\kappa)}\right)^{T}\right.$, hence we have $x^{T} \mathcal{Q}_{2, k}^{I,(\kappa)} x=0 \forall x \in$ $\left.\mathbb{R}^{N}\right)$. 
Then the

$$
\begin{aligned}
& \text { LHS of (63) } \\
= & (\cos \boldsymbol{\theta}-\jmath \sin \boldsymbol{\theta})^{T}\left(\mathcal{Q}_{2, k}^{R,(\kappa)}+\jmath \mathcal{Q}_{2, k}^{I,(\kappa)}\right)(\cos \boldsymbol{\theta}+\jmath \sin \boldsymbol{\theta}) \\
= & \left((\cos \boldsymbol{\theta})^{T} \mathcal{Q}_{2, k}^{R,(\kappa)}-\jmath(\sin \boldsymbol{\theta})^{T} \mathcal{Q}_{2, k}^{R,(\kappa)}+\jmath(\cos \boldsymbol{\theta})^{T} \mathcal{Q}_{2, k}^{I,(\kappa)}\right. \\
& \left.-(\sin \boldsymbol{\theta})^{T} \mathcal{Q}_{2, k}^{I,(\kappa)}\right)(\cos \boldsymbol{\theta}+\jmath \sin \boldsymbol{\theta}) \\
= & (\cos \boldsymbol{\theta})^{T} \mathcal{Q}_{2, k}^{R,(\kappa)} \cos \boldsymbol{\theta}-\jmath(\sin \boldsymbol{\theta})^{T} \mathcal{Q}_{2, k}^{R,(\kappa)} \cos \boldsymbol{\theta} \\
& +\jmath(\cos \boldsymbol{\theta})^{T} \mathcal{Q}_{2, k}^{I,(\kappa)} \cos \boldsymbol{\theta}-(\sin \boldsymbol{\theta})^{T} \mathcal{Q}_{2, k}^{I,(\kappa)} \cos \boldsymbol{\theta} \\
& +\jmath(\cos \boldsymbol{\theta})^{T} \mathcal{Q}_{2,(\kappa)}^{R,(\kappa)} \sin \boldsymbol{\theta}+(\sin \boldsymbol{\theta})^{T} \mathcal{Q}_{2,(, k)}^{R,(\kappa)} \sin \boldsymbol{\theta} \\
& -(\cos \boldsymbol{\theta})^{T} \mathcal{Q}_{2, k}^{I,(\kappa)} \sin \boldsymbol{\theta}-\jmath(\sin \boldsymbol{\theta})^{T} \mathcal{Q}_{2, k}^{I,(\kappa)} \sin \boldsymbol{\theta} \\
= & (\cos \boldsymbol{\theta})^{T} \mathcal{Q}_{2, k}^{R,(\kappa)} \cos \boldsymbol{\theta}-(\sin \boldsymbol{\theta})^{T} \mathcal{Q}_{2, k}^{I,(\kappa)} \cos \boldsymbol{\theta} \\
& +(\sin \boldsymbol{\theta})^{T} \mathcal{Q}_{2, k}^{R,(\kappa)} \sin \boldsymbol{\theta}-(\cos \boldsymbol{\theta})^{T} \mathcal{Q}_{2, k}^{I,(\kappa)} \sin \boldsymbol{\theta} \\
= & \text { RHS of }(63),
\end{aligned}
$$

proving (63).

Furthermore,

$$
\begin{aligned}
& \text { LHS of (64) } \\
= & 2 \Re\left\{\left(e^{\jmath \boldsymbol{\theta}}\right)^{T} \mathcal{Q}_{12, k}^{(\kappa)} e^{\jmath \boldsymbol{\theta}}\right\} \\
= & 2 \Re\left\{(\cos \boldsymbol{\theta}+\jmath \sin \boldsymbol{\theta})^{T}\left(\mathcal{Q}_{1, k}^{R,(\kappa)}+\jmath \mathcal{Q}_{1, k}^{I,(\kappa)}\right)\right. \\
& (\cos \boldsymbol{\theta}+\jmath \sin \boldsymbol{\theta})\} \\
= & 2\left((\cos \boldsymbol{\theta})^{T} \mathcal{Q}_{1, k}^{R,(\kappa)} \cos \boldsymbol{\theta}-(\cos \boldsymbol{\theta})^{T} \mathcal{Q}_{1, k}^{I,(\kappa)} \sin \boldsymbol{\theta}\right. \\
& \left.-(\sin \boldsymbol{\theta})^{T} \mathcal{Q}_{1, k}^{R,(\kappa)} \sin \boldsymbol{\theta}-(\sin \boldsymbol{\theta})^{T} \mathcal{Q}_{1, k}^{I,(\kappa)} \cos \boldsymbol{\theta}\right) \\
= & \text { RHS of }(64),
\end{aligned}
$$

proving (64).

\section{REFERENCES}

[1] H. H. M. Tam, H. D. Tuan, and D. T. Ngo, "Successive convex quadratic programming for quality-of-service management in full-duplex MUMIMO multicell networks," IEEE Trans. Commun., vol. 64, pp. 23402353, June 2016.

[2] L. D. Nguyen, H. D. Tuan, T. Q. Duong, H. V. Poor, and L. Hanzo, "Energy-efficient multi-cell massive mimo subject to minimum userrate constraints," IEEE Transactions on Communications, vol. 69, no. 2, pp. 914-928, 2021.

[3] T. Yoo and A. Goldsmith, "On the optimality of multiantenna broadcast scheduling using zero-forcing beamforming," IEEE J. Sel. Areas Commun., vol. 54, pp. 528-541, Mar. 2006.

[4] L. D. Nguyen, H. D. Tuan, T. Q. Duong, O. A. Dobre, and H. V. Poor, "Downlink beamforming for energy-efficient heterogeneous networks with massive MIMO and small cells," IEEE Trans. Wirel. Commun., vol. 17, pp. 3386-3400, May 2018.

[5] L. D. Nguyen, H. D. Tuan, T. Q. Duong, and H. V. Poor, "Multi-user regularized zero-forcing beamforming," IEEE Trans. Signal Process., vol. 67, pp. 2839-2853, Jun. 2019.

[6] A. A. Nasir, H. D. Tuan, T. Q. Duong, H. V. Poor, and L. Hanzo, "Hybrid beamforming for multi-user millimeter-wave networks," IEEE Trans. Vehic. Techn., vol. 69, pp. 2943-2956, Mar. 2020.

[7] S. Tretyakov, V. Asadchy, and A. Diaz-Rubio, "Chapter 6: Metasurfaces for general control of reflection and transmission," in World Scientific Handbook of Metamaterials and Plasmonics, pp. 249-293, 2017.

[8] C. Huang, A. Zappone, G. C. Alexandropoulos, M. Debbah, and C. Yuen, "Reconfigurable intelligent surfaces for energy efficiency in wireless communication," IEEE Trans. Wirel. Commun., vol. 18, pp. 4157-4170, Aug. 2019.

[9] Q. Wu and R. Zhang, "Towards smart and reconfigurable environment: Intelligent reflecting surface aided wireless network," IEEE Commun. Mag., pp. 1-7, 2019.

[10] M. Di Renzo, M. Debbah, D.-T. Phan-Huy, A. Zappone, M.-S. Alouini, C. Yuen, V. Sciancalepore, G. C. Alexandropoulos, J. Hoydis, H. Gacanin, J. de Rosny, A. Bounceu, G. Lerosey, and M. Fink, "Smart radio environments empowered by AI reconfigurable meta-surfaces: An idea whose time has come," EURASIP J. Wirel. Commun. Network., no. 1 , p. $129,2019$.
[11] C. Huang, S. Hu, G. C. Alexandropoulos, A. Zappone, C. Yuen, R. Zhang, M. D. Renzo, and M. Debbah, "Holographic MIMO surfaces for $6 \mathrm{G}$ wireless networks: Opportunities, challenges, and trends," IEEE Wirel. Commun., vol. 27, no. 5, pp. 118-125, 2020.

[12] L. Yang, F. Meng, J. Zhang, M. O. Hasna, and M. D. Renzo, "On the performance of ris-assisted dual-hop uav communication systems," IEEE Trans. Vehic. Techn., vol. 69, no. 9, pp. 10385-10390, 2020.

[13] X. Liu, Y. Liu, and Y. Chen, "Machine learning empowered trajectory and passive beamforming design in uav-ris wireless networks," IEEE $J$. Selec. Areas Commun., pp. 1-1, 2020.

[14] S. Li, B. Duo, X. Yuan, Y. Liang, and M. Di Renzo, "Reconfigurable intelligent surface assisted uav communication: Joint trajectory design and passive beamforming," IEEE Wireless Commun. Lett., vol. 9, no. 5, pp. 716-720, 2020.

[15] L. Yang, X. Yan, D. B. da Costa, T. A. Tsiftsis, H. C. Yang, and M. S. Alouini, "Indoor mixed dual-hop vlc/rf systems through reconfigurable intelligent surfaces," IEEE Wirel. Commun. Lett., vol. 9, no. 11, pp. 1995-1999, 2020.

[16] L. Wei, C. Huang, G. C. Alexandropoulos, C. Yuen, Z. Zhang, and M. Debbah, "Channel estimation for RIS-empowered multi-user MISO wireless communications," IEEE Tran. Commun. (early access).

[17] J. He, H. Wymeersch, and M. Juntti, "Channel estimation for risaided mmwave mimo systems via atomic norm minimization," IEEE Transactions on Wireless Communications, pp. 1-1, 2021.

[18] Z. Zhang, C. Zhang, C. Jiang, F. Jia, J. Ge, and F. Gong, "Improving physical layer security for reconfigurable intelligent surface aided noma $6 \mathrm{~g}$ networks," IEEE Transactions on Vehicular Technology, pp. 1-1, 2021.

[19] M. H. Khoshafa, T. M. N. Ngatched, and M. H. Ahmed, "Reconfigurable intelligent surfaces-aided physical layer security enhancement in $\mathrm{d} 2 \mathrm{~d}$ underlay communications," IEEE Commun. Lett., pp. 1-1, 2020.

[20] C. Huang, R. Mo, and C. Yuen, "Reconfigurable intelligent surface assisted multiuser MISO systems exploiting deep reinforcement learning," IEEE J. Select. Areas Commun., vol. 38, no. 8, pp. 1839-1850, 2020.

[21] Q. U. A. Nadeem, A. Kammoun, A. Chaaban, M. Debbah, and M. S. Alouini, "Asymptotic max-min SINR analysis of reconfigurable intelligent surface assisted MISO systems," IEEE Trans. Wirel. Commun., vol. 19, no. 12, pp. 7748-7764, 2020.

[22] Q. Wu and R. Zhang, "Intelligent reflecting surface enhanced wireless network via joint active and passive beamforming," IEEE Trans. Wirel. Commun., vol. 18, pp. 5394-5409, Nov 2019.

[23] G. Zhou, C. Pan, H. Ren, K. Wang, W. Xu, and A. Nallanathan, "Intelligent reflecting surface aided multigroup multicast MISO communication systems," IEEE Trans. Signal Process., vol. 68, pp. 3236-3251, 2020.

[24] C. Pan, H. Ren, K. Wang, W. Xu, M. Elkashlan, A. Nallanathan, and L. Hanzo, "Multicell MIMO communications relying on intelligent reflecting surface," IEEE Trans Wirel. Commun., vol. 19, pp. 5218-5233, Aug. 2020.

[25] C. Pan, H. Ren, K. Wang, M. Elkashlan, A. Nallanathan, J. Wang, and L. Hanzo, "Intelligent reflecting surface aided MIMO broadcasting for simultaneous wireless information and power transfer," IEEE J. Sel. Areas Commun., vol. 38, pp. 1719-1734, Aug. 2020.

[26] M. M. Zhao, Q. Wu, M. J. Zhao, and R. Zhang, "Intelligent reflecting surface enhanced wireless networks: Two-timescale beamforming optimization," IEEE Trans. Wirel. Commun., vol. 20, no. 1, pp. 2-17, 2021.

[27] H. Yu, H. D. Tuan, A. A. Nasir, T. Q. Duong, and H. V. Poor, "Joint design of reconfigurable intelligent surfaces and transmit beamforming under proper and improper Gaussian signaling," IEEE J. Sel. Areas Commun., vol. 38, pp. 2589-2603, Nov. 2020.

[28] S. Lagen, A. Agustin, and J. Vidal, "Coexisting linear and widely linear transceivers in the MIMO interference channel," IEEE Trans. Signal Process., vol. 64, pp. 652-664, Feb 2016.

[29] A. A. Nasir, H. D. Tuan, T. Q. Duong, and H. V. Poor, "Improper Gaussian signaling for broadcast interference networks," IEEE Signal Process. Lett., vol. 26, pp. 808-812, Jun. 2019.

[30] H. D. Tuan, A. A. Nasir, H. H. Nguyen, T. Q. Duong, and H. V. Poor, "Non-orthogonal multiple access with improper Gaussian signaling," IEEE J. Selec. Topics Signal Process., vol. 13, pp. 496-507, Mar. 2019.

[31] H. Yu, H. D. Tuan, T. Q. Duong, and L. Hanzo, "Improper Gaussian signaling for integrated data and energy networking," IEEE Trans. Commun., vol. 68, pp. 3922-3934, Jun. 2020.

[32] H. Yu, H. D. Tuan, A. A. Nasir, T. Q. Duong, and L. Hanzo, "Improper gaussian signaling for computationally tractable energy and information beamforming," IEEE Trans. Vehic. Techn., vol. 69, no. 11, pp. 1399013995, 2020. 
[33] O. Ozdogan, E. Bjornson, and E. G. Larsson, "Intelligent reflecting surfaces: Physics, propagation, and pathloss modeling," https://arxiv.org/abs/1911.03359.

[34] E. Bjornson, O. Ozdogan, and E. G. Larsson, "Intelligent reflecting surface versus decode-and-forward: How large surfaces are needed to beat relaying?," IEEE Wirel. Commun. Lett., vol. 9, no. 2, pp. 244-248, 2020.

[35] Q.-U.-A. Nadeem, A. Kammoun, M. Debbah, and M.-S. Alouini, "A generalized spatial correlation model for 3D MIMO channels based on the Fourier coefficients of power spectrums," IEEE Trans. Signal Process., vol. 63, pp. 3671-3686, Jul. 2015.

[36] P. D. Tao and L. T. H. An, "A d. c. optimization algorithm for solving the trust-region subproblem," SIAM J. Optimiz., vol. 8, no. 2, pp. 476-505, 1998.

[37] A. Migdalas, "A regularization of the Frank-and-Wolfe method and unification of certain nonlinear programming methods," Math. Program. vol. 65, pp. 331-346, 1994.

[38] P. J. Schrerier and L. L. Scharf, Statistical Signal Processing of ComplexValued Data: The Theory of Improper and Noncircular Signals. Cambridge University Press, 2010.

[39] I. E. Telatar, "Capacity of multi-antenna Gaussian channels," Eur. Trans. Telecommun., vol. 10, pp. 585-595, Nov./Dec. 1999.

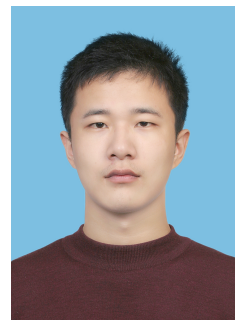

Hongwen Yu received the B.S. degree in Communication and Information Engineering from the Shanghai University, Shanghai, China, in 2011, and the M.S. degree in Communication and Information Engineering from the Shanghai University, Shanghai, China, in 2014. He is currently pursuing the Ph.D. degree with the School of Electrical and Data Engineering, University of Technology Sydney, Ultimo, NSW, Australia, and with Shanghai University, Shanghai, China. His current research interests include optimization methods for wireless communication and signal processing.

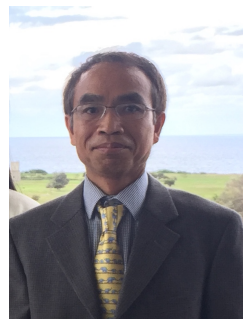

Hoang Duong Tuan received the Diploma (Hons.) and Ph.D. degrees in applied mathematics from Odessa State University, Ukraine, in 1987 and 1991, respectively. He spent nine academic years in Japan as an Assistant Professor in the Department of Electronic-Mechanical Engineering, Nagoya University, from 1994 to 1999, and then as an Associate Professor in the Department of Electrical and Computer Engineering, Toyota Technological Institute, Nagoya, from 1999 to 2003. He was a Professor with the School of Electrical Engineering and Telecommunications, University of New South Wales, from 2003 to 2011. He is currently a Professor with the School of Electrical and Data Engineering, University of Technology Sydney. He has been involved in research with the areas of optimization, control, signal processing, wireless communication, and biomedical engineering for more than 20 years.

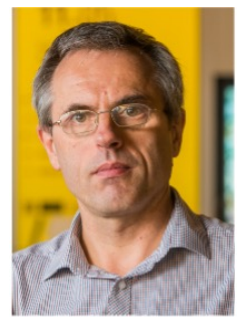

Eryk Dutkiewicz received his B.E. degree in Electrical and Electronic Engineering from the University of Adelaide in 1988, his M.Sc. degree in Applied Mathematics from the University of Adelaide in 1992 and his $\mathrm{PhD}$ in Telecommunications from the University of Wollongong in 1996. His industry experience includes management of the Wireless Research Laboratory at Motorola in early 2000's. Prof. Dutkiewicz is currently the Head of School of Electrical and Data Engineering at the University of Technology Sydney, Australia. He is a Senior Member of IEEE. He also holds a professorial appointment at Hokkaido University in Japan. His current research interests cover 5G/6G and IoT networks.

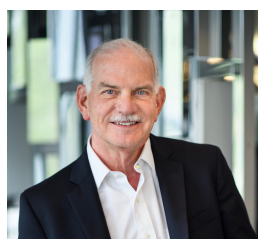

H. Vincent Poor (S72, M77, SM82, F87) received the Ph.D. degree in EECS from Princeton University in 1977. From 1977 until 1990, he was on the faculty of the University of Illinois at Urbana-Champaign. Since 1990 he has been on the faculty at Princeton, where he is currently the Michael Henry Strater University Professor. During 2006 to 2016, he served as the dean of Princeton's School of Engineering and Applied Science. He has also held visiting appointments at several other universities, including most recently at Berkeley and Cambridge. His research interests are in the areas of information theory, machine learning and network science, and their applications in wireless networks, energy systems and related fields. Among his publications in these areas is the forthcoming book Machine Learning and Wireless Communications (Cambridge University Press, 2021).

Dr. Poor is a member of the National Academy of Engineering and the National Academy of Sciences and is a foreign member of the Chinese Academy of Sciences, the Royal Society, and other national and international academies. Recent recognition of his work includes the 2017 IEEE Alexander Graham Bell Medal and a D.Eng. honoris causa from the University of Waterloo awarded in 2019.

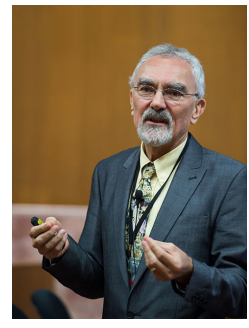

Lajos Hanzo (http://www-mobile.ecs.soton.ac.uk, https://en.wikipedia.org/wiki/Lajos_Hanzo) (FIEEE'04) received his Master degree and Doctorate in 1976 and 1983, respectively from the Technical University (TU) of Budapest. He was also awarded the Doctor of Sciences (DSc) degree by the University of Southampton (2004) and Honorary Doctorates by the TU of Budapest (2009) and by the University of Edinburgh (2015). He is a Foreign Member of the Hungarian Academy of Sciences and a former Editor-in-Chief of the IEEE Press. He has served several terms as Governor of both IEEE ComSoc and of VTS. He has published 2000+ contributions at IEEE Xplore, 19 Wiley-IEEE Press books and has helped the fast-track career of $123 \mathrm{PhD}$ students. Over 40 of them are Professors at various stages of their careers in academia and many of them are leading scientists in the wireless industry. He is also a Fellow of the Royal Academy of Engineering (FREng), of the IET and of EURASIP. 